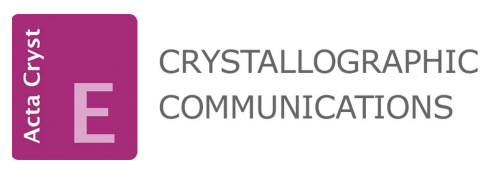

ISSN 2056-9890

Received 4 February 2016

Accepted 16 February 2016 Energía Atómica, Argentina

Keywords: crystal structure; cisplatin; platinum(II); benzothiazole; anticancer.

CCDC references: $1441324 ; 1441327$; $1441326 ; 1441325$

Supporting information: this article has supporting information at journals.iucr.org/e

\section{New platinum(II) complexes with benzothiazole ligands}

\author{
José A. Carmona-Negrón, ${ }^{a}$ Mayra E. Cádiz, ${ }^{a}$ Curtis E. Moore, ${ }^{b}$ Arnold L. Rheingold ${ }^{b}$ \\ and Enrique Meléndez ${ }^{a *}$
}

Edited by R. F. Baggio, Comisión Nacional de

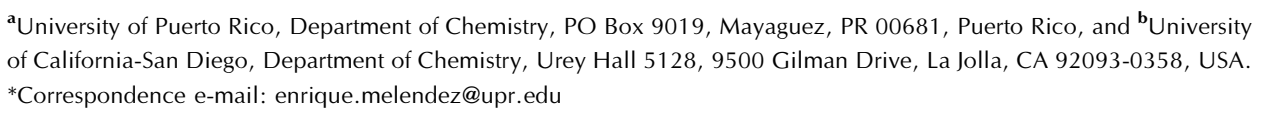

Four new platinum(II) complexes, namely tetraethylammonium tribromido(2-methyl-1,3-benzothiazole- $\kappa N)$ platinate(II), $\quad\left[\mathrm{NEt}_{4}\right]\left[\mathrm{PtBr}_{3}\left(\mathrm{C}_{8} \mathrm{H}_{7} \mathrm{NS}\right)\right] \quad$ (1), tetraethylammonium tribromido(6-methoxy-2-methyl-1,3-benzothiazole- $\kappa N)$ platinate(II), $\left[\mathrm{NEt}_{4}\right]\left[\mathrm{PtBr}_{3}\left(\mathrm{C}_{9} \mathrm{H}_{9} \mathrm{NOS}\right)\right]$ (2), tetraethylammonium tribromido(2,5,6-trimethyl-1,3-benzothiazole- $\kappa N)$ platinate(II), $\left[\mathrm{NEt}_{4}\right]\left[\mathrm{PtBr}_{3}\left(\mathrm{C}_{10} \mathrm{H}_{11} \mathrm{NS}\right)\right]$ (3), and tetraethylammonium tribromido(2-methyl-5-nitro-1,3-benzothiazole$\kappa N)$ platinate(II), $\left[\mathrm{NEt}_{4}\right]\left[\mathrm{PtBr}_{3}\left(\mathrm{C}_{8} \mathrm{H}_{6} \mathrm{~N}_{2} \mathrm{O}_{2} \mathrm{~S}\right)\right](4)$, have been synthesized and structurally characterized by single-crystal X-ray diffraction techniques. These species are precursors of compounds with potential application in cancer chemotherapy. All four platinum(II) complexes adopt the expected squareplanar coordination geometry, and the benzothiazole ligand is engaged in bonding to the metal atom through the imine $\mathrm{N}$ atom $(\mathrm{Pt}-\mathrm{N})$. The $\mathrm{Pt}-\mathrm{N}$ bond lengths are normal: 2.035 (5), 2.025 (4), 2.027 (5) and 2.041 (4) $\AA$ for complexes $\mathbf{1}, \mathbf{2}, 3$ and 4 , respectively. The benzothiazole ligands are positioned out of the square plane, with dihedral angles ranging from 76.4 (4) to 88.1 (4) ${ }^{\circ}$. $\mathrm{The} \mathrm{NEt}_{4}$ cation in $\mathbf{3}$ is disordered with $0.57 / 0.43$ occupancies.

\section{Chemical context}

The synthesis of new platinum complexes as potential drugs for cancer is still of interest for medicinal chemists. The structural details of these complexes provide the opportunity to predict, to a certain extent, the potential biological activity of these species. In this regard, four new platinum(II) complexes with benzothiazole ligands of general formula $\left[\mathrm{PtBr}_{3} L\right]^{-}$have been synthesized according to the equation below and their structures characterized.

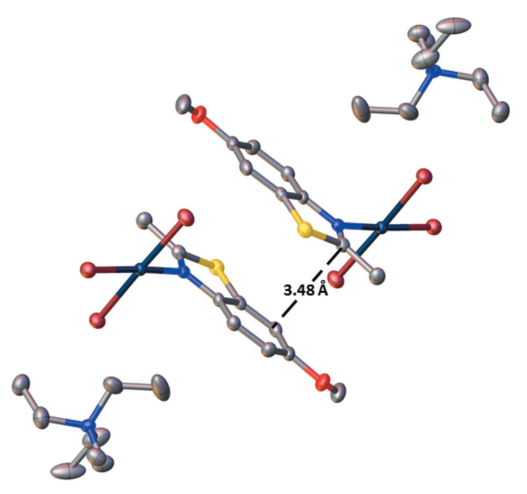

$\left[\mathrm{NEt}_{4}\right]_{2}\left[\mathrm{Pt}_{2} \mathrm{Br}_{6}\right]+2 L \rightarrow 2\left[\mathrm{NEt}_{4}\right]\left[\mathrm{PtBr}_{3} L\right]$

$L=$ 2-methyl-1,3-benzothiazole (1), 6-methoxy-2-methyl-1,3benzothiazole (2), 2,5,6-trimethyl-1,3-benzothiazole (3), and 2-methyl-5-nitro-1,3-benzothiazole (4). All complexes showed the benzothiazoles to coordinate the $\mathrm{Pt}^{\mathrm{II}}$ atom through the imino nitrogen atom. Also, the benzothiazole is positioned out of the square plane with dihedral angles between 76.4 (4) and $88.1(4)^{\circ}$, as previously reported in other platinum-benzothiazole complexes. Given that benzothiazoles have anticancer properties, these platinum complexes may have enhanced properties as a result of potential synergism between the ligand and $\mathrm{Pt}^{\mathrm{II}}$. This deserves further studies as suggested by Noolvi et al. (2012) 


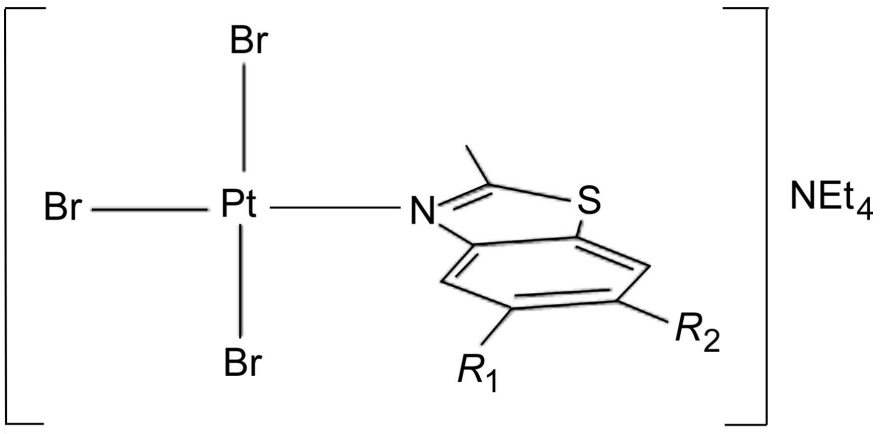

$1 R_{1}=R_{2}=\mathrm{H} ; \quad 2 R_{1}=\mathrm{H}, \quad R_{2}=\mathrm{OCH}_{3}$

$3 R_{1}=R_{2}=\mathrm{CH}_{3} ; 4 R_{1}=\mathrm{NO}_{2}, R_{2}=\mathrm{H}$

\section{Structural commentary}

To elucidate with certainty and accurately the platinum coordination patterns, the structural determination of the complexes was performed by single crystal X-ray diffraction technique. Table 1 contains selected bond lengths, dihedral angles and torsion angles. All of the title complexes adopt a square-planar coordination geometry about the $\mathrm{Pt}^{\mathrm{II}}$ atom with a deviation of no more than $4^{\circ}$ from ideal $180^{\circ}$ and $90^{\circ}$ angles. As reported previously, although not predicted using Pearson's hard-soft acid base theory, the benzothiazole is engaged in bonding to the metal through the imine nitrogen $(\mathrm{Pt}-\mathrm{N})$ instead of Pt-S coordination (Muir et al., 1987, 1988a,b, 1990; Gomez et al., 1988; Lozano et al., 1994). Also the benzothia-

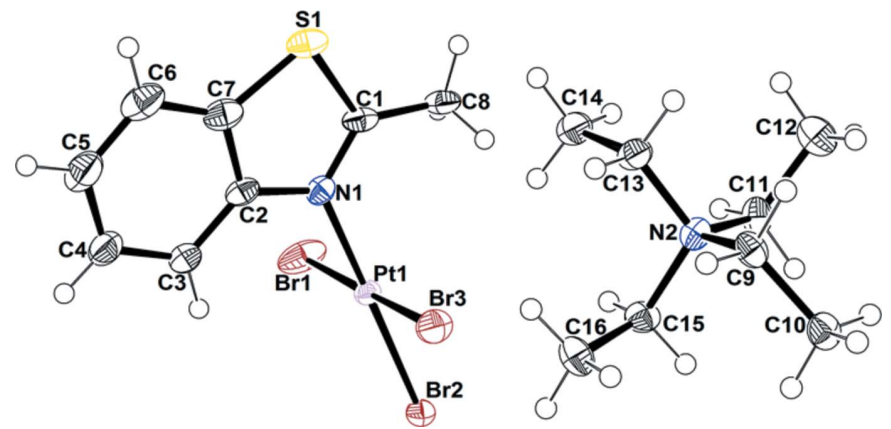

Figure 1

The molecular structure of $\left[\mathrm{NEt}_{4}\right]\left[\mathrm{PtBr}_{3}(2-\mathrm{Me}\right.$-benzothiazole $\left.)\right]$ (1), with displacement ellipsoids drawn at the $50 \%$ probability level.

zole ligand is positioned out of the square plane as discussed below.

Figs. 1-4 show the molecular structures of the four new complexes. [ $\left.\mathrm{NEt}_{4}\right]\left[\mathrm{PtBr}_{3}(2-\mathrm{Me}\right.$-benzothiazole)] (1) crystallizes in an orthorhombic unit cell with eight formula units. It is a square-planar complex with $\mathrm{Pt}-\mathrm{N}$ and average $\mathrm{Pt}-\mathrm{Br}$ bond lengths of 2.035 (5) and 2.433 (6) $\AA$, respectively, which are within the expected range for $\mathrm{Pt}^{\mathrm{II}}$ complexes. There is no trans-influence observed in the $\mathrm{Pt}-\mathrm{Br}$ bond trans to the $\mathrm{Pt}-\mathrm{N}$ bond. The benzothiazole ligand is planar and the methyl group resides in the ligand plane. The dihedral angle between the $\mathrm{PtBr}_{3} \mathrm{~N}$ unit and the benzothiazole ring is $88.1(4)^{\circ}$, similar to those observed in other $\mathrm{Pt}^{\mathrm{II}}$-benzothiazole complexes, as a result of reducing the steric strain between $\mathrm{PtBr}_{3}$ and the benzothiazole ligand (Muir et al., 1987, 1988a,b, 1990; Gomez et al., 1988; Lozano et al., 1994). Two types of $\mathrm{N}-\mathrm{C}$ bonds are present, one long $[\mathrm{N}-\mathrm{C} 21.408(7) \AA]$ and one short $[\mathrm{N}-\mathrm{C} 1$

Table 1

Selected bond distances and angles $\left(\AA{ }^{\circ}\right)$.

The dihedral angle is between the $\mathrm{Pt}-\mathrm{Br}_{3} \mathrm{~N}$ unit and the benzothiazole ring. The torsion angle is between the benzothiazole ring and the $R$ group.

\begin{tabular}{|c|c|c|c|c|}
\hline & 1 & 2 & 3 & 4 \\
\hline $\mathrm{Pt}-\mathrm{Br}_{\text {average }}$ & $2.433(6)$ & $2.430(6)$ & $2.425(6)$ & $2.431(8)$ \\
\hline $\mathrm{Pt}-\mathrm{N}$ & $2.035(5)$ & $2.025(4)$ & $2.027(5)$ & $2.041(4)$ \\
\hline $\mathrm{N} 1-\mathrm{C} 2$ & $1.408(7)$ & $1.396(6)$ & $1.401(8)$ & $1.383(6)$ \\
\hline $\mathrm{Pt}-\mathrm{Br} 1$ & $2.4375(8)$ & $2.4352(5)$ & $2.4309(7)$ & $2.4335(6)$ \\
\hline $\mathrm{Pt}-\mathrm{Br} 2$ & $2.4349(8)$ & $2.4241(7)$ & $2.4198(7)$ & $2.4216(5)$ \\
\hline $\mathrm{Pt}-\mathrm{Br} 3$ & $2.4268(7)$ & $2.4309(5)$ & $2.4240(7)$ & $2.4367(5)$ \\
\hline $\mathrm{C} 1-\mathrm{N} 1-\mathrm{C} 2$ & $113.0(5)$ & $112.6(4)$ & $112.3(5)$ & $111.9(4)$ \\
\hline $\mathrm{C} 1-\mathrm{S}-\mathrm{C} 7$ & $90.3(3)$ & $89.9(2)$ & $89.8(3)$ & $90.0(2)$ \\
\hline $\mathrm{N} 1-\mathrm{Pt}-\mathrm{Br} 1$ & $90.6(1)$ & $87.0(1)$ & $89.2(1)$ & $88.6(1)$ \\
\hline $\mathrm{N} 1-\mathrm{Pt}-\mathrm{Br} 3$ & $86.4(1)$ & $89.3(1)$ & $88.5(1)$ & $89.3(1)$ \\
\hline $\mathrm{N} 1-\mathrm{Pt}-\mathrm{Br} 2$ & $177.7(1)$ & $177.4(1)$ & $178.8(1)$ & $178.4(1)$ \\
\hline $\mathrm{Br} 1-\mathrm{Pt}-\mathrm{Br} 3$ & $176.85(2)$ & $176.30(2)$ & $177.45(3)$ & $176.23(2)$ \\
\hline $\mathrm{Br} 2-\mathrm{Pt}-\mathrm{Br} 3$ & $91.69(2)$ & $92.51(2)$ & $91.23(2)$ & $91.18(2)$ \\
\hline \multirow[t]{3}{*}{ Torsion angle } & $0.72(1)\left(\mathrm{CH}_{3}\right)$ & $11.9(7)\left(\mathrm{OCH}_{3}\right)$ & $1.5(5)\left(\mathrm{C}_{8} \mathrm{H}_{3}\right)$ & $1.1(5)\left(\mathrm{CH}_{3}\right)$ \\
\hline & & & $0.2(6)\left(\mathrm{C}_{9} \mathrm{H}_{3}\right)$ & $7.5(7)\left(\mathrm{NO}_{2}\right)$ \\
\hline & & & $0.3(6)\left(\mathrm{C}_{10} \mathrm{H}_{3}\right)$ & \\
\hline
\end{tabular}




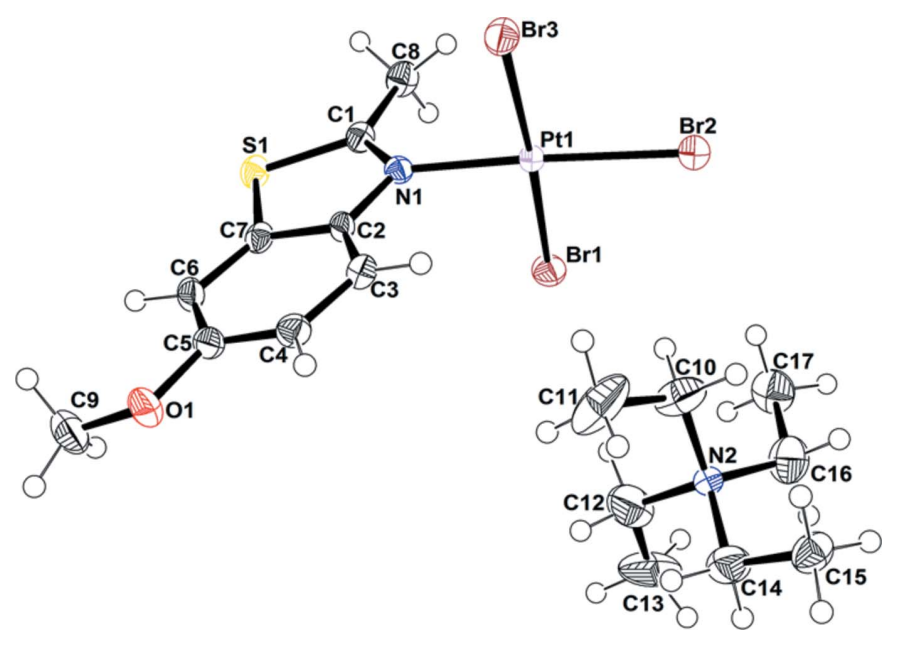

Figure 2

The molecular structure of [ $\left.\mathrm{NEt}_{4}\right]\left[\mathrm{PtBr}_{3}(6-\mathrm{OMe}-2-\mathrm{Me}\right.$-benzothiazole $\left.)\right]$ (2), with displacement ellipsoids drawn at the $50 \%$ probability level.
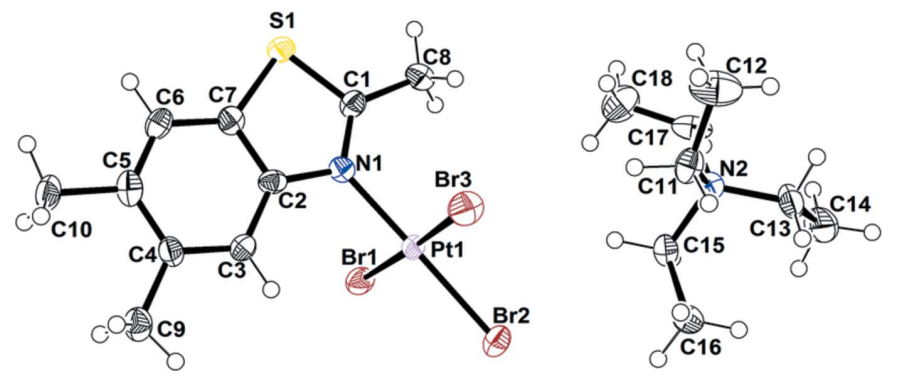

Figure 3

The molecular structure of $\left[\mathrm{NEt}_{4}\right]\left[\mathrm{PtBr}_{3}(2,5,6-\mathrm{Me}\right.$-benzothiazole $\left.)\right](\mathbf{3})$, with displacement ellipsoids drawn at the $50 \%$ probability level. The $\mathrm{NEt}_{4}$ cation in 3 presented disorder with $0.57 / 0.43$ occupancies. Only the major fraction is shown for clarity.

1.309 (7) $\mathrm{A}$ ], indicating the presence of single- and doublebond character in the thiazole ring. The angle at the $\mathrm{S}$ atom in the thiazole ring is $90.3(3)^{\circ}$ suggesting it is using unhybridized $p$ orbitals for bonding.

$\left[\mathrm{NEt}_{4}\right]\left[\mathrm{PtBr}_{3}(6-\mathrm{OMe}-2-\mathrm{Me}-\right.$ benzothiazole $\left.)\right] \quad(2), \quad\left[\mathrm{NEt}_{4}\right]-$ $\left[\mathrm{PtBr}_{3}(2,5,6-\mathrm{Me}\right.$-benzothiazole $\left.)\right](3)$ and $\left[\mathrm{NEt}_{4}\right]\left[\mathrm{PtBr}_{3}\left(5-\mathrm{NO}_{2}-\right.\right.$ 2-Me-benzothiazole)] (4) crystallize in the same type of unit cell and space group, monoclinic $P 2_{1} / n$, containing four
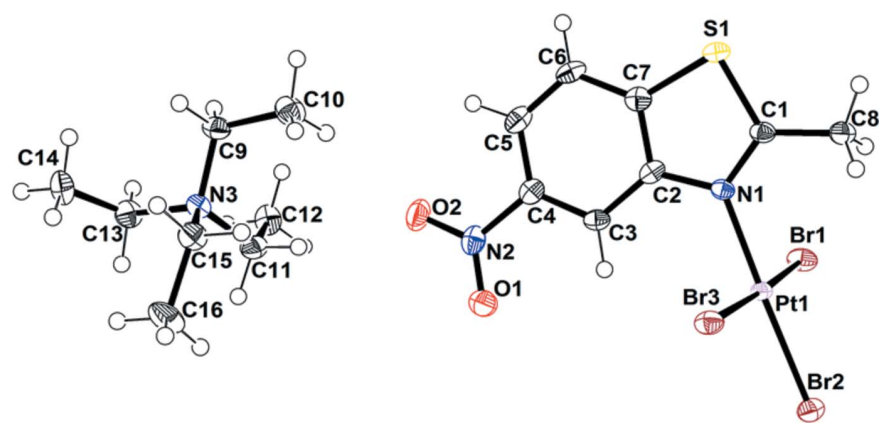

Figure 4

The molecular structure of $\left[\mathrm{NEt}_{4}\right]\left[\mathrm{PtBr}_{3}\left(5-\mathrm{NO}_{2}-2-\mathrm{Me}\right.\right.$-benzothiazole $\left.)\right]$ (4), with displacement ellipsoids drawn at the $50 \%$ probability level. formula units. The $\mathrm{Pt}-\mathrm{N}$ and average $\mathrm{Pt}-\mathrm{Br}$ bond lengths for $\mathbf{2 , 3}$, and $\mathbf{4}$ are $2.025(4) / 2.430$ (6) $\AA, 2.027$ (5)/2.425 (6) $\AA$ and $2.041(4) / 2.431(8) \AA$, respectively, which are within the expected range. The dihedral angle between $\operatorname{PtBr}_{3} \mathrm{~N}$ and the benzothiazole in $\mathbf{2}$ is $86.7(3)^{\circ}$ and the torsion angle between the aromatic ring and the $\mathrm{OCH}_{3}$ group is $11.9(7)^{\circ}$. The $\mathrm{C}-\mathrm{O}$ $\left(\mathrm{OCH}_{3}\right)$ bond length is $1.427(7) \AA$, and the $\mathrm{C}-\mathrm{O}-\mathrm{CH}_{3}$ angle is $116.3(5)^{\circ}$. In contrast to $\mathbf{1}$ and $\mathbf{2},\left[\mathrm{NEt}_{4}\right]\left[\mathrm{PtBr}_{3}(2,5,6-\mathrm{Me}-\right.$ benzothiazole $)]$ and $\left[\mathrm{NEt}_{4}\right]\left[\mathrm{PtBr}_{3}\left(5-\mathrm{NO}_{2}\right.\right.$-2-Me-benzothiazole)] have lower dihedral angles between the $\mathrm{PtBr}_{3} \mathrm{~N}$ unit and the benzothiazole ring, $78.6(4)$ and 76.(4) ${ }^{\circ}$, respectively. The methyl groups on $\mathbf{3}$ and $\mathbf{4}$ are almost co-planar with the benzothiazole plane with deviations $\leq 1.60^{\circ}$ but in $\mathbf{4}$, the $\mathrm{NO}_{2}$ group is out of the benzothiazole plane with a torsion angle of
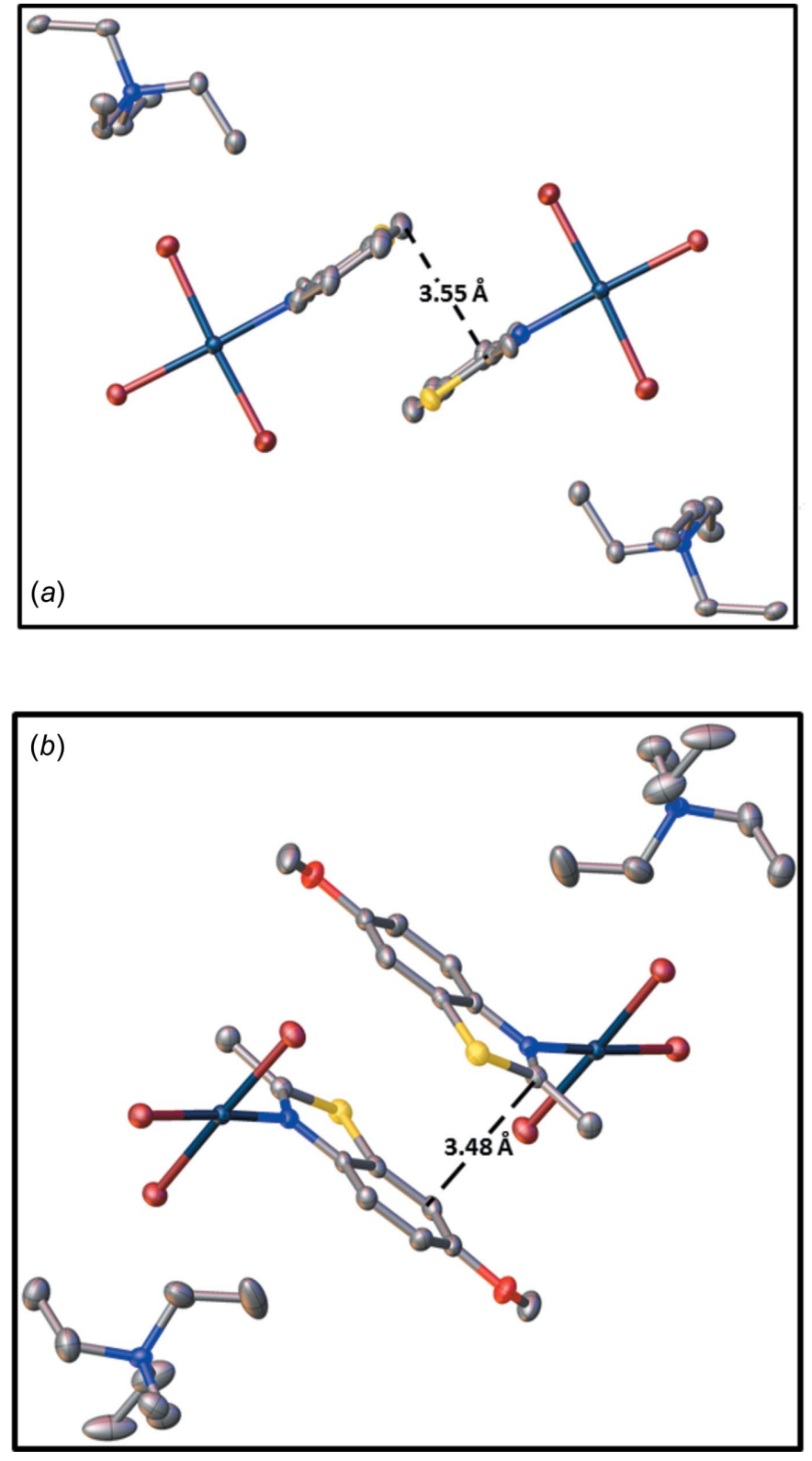

Figure 5

Details of the packing interactions in $(a)\left[\mathrm{NEt}_{4}\right]\left[\mathrm{PtBr}_{3}(2-\mathrm{Me}\right.$-benzothiazole)] and $(b)\left[\mathrm{NEt}_{4}\right]\left[\mathrm{PtBr}_{3}(6-\mathrm{Ome}-2-\mathrm{Me}-\right.$ benzothiazole $\left.)\right]$. 
Table 2

Experimental details.

(1)

Crystal data

Chemical formula

$M_{\mathrm{r}}$

Crystal system,

space group

Temperature (K)

$a, b, c(\AA)$

$\alpha, \beta, \gamma\left({ }^{\circ}\right)$

$V\left(\AA^{3}\right)$

$Z$

Radiation type

$\mu\left(\mathrm{mm}^{-1}\right)$

Crystal size (mm)

Data collection

Diffractometer

Absorption correction

$T_{\min }, T_{\max }$

No. of measured, independent and

observed $[I>2 \sigma(I)]$

reflections

$R_{\text {int }}$

$(\sin \theta / \lambda)_{\max }\left(\AA^{-1}\right)$

0.047

$\left(\mathrm{C}_{8} \mathrm{H}_{20} \mathrm{~N}\right)\left[\mathrm{PtBr}_{3}\left(\mathrm{C}_{8} \mathrm{H}_{7} \mathrm{NS}\right)\right]$

714.27

Orthorhombic, $\mathrm{Pbca}$

100

12.114 (3), 10.656 (3), $34.043(9)$

90, 90,90

4394 (2)

8

Mo $K \alpha$

11.94

$0.18 \times 0.16 \times 0.12$

(2)

$\left(\mathrm{C}_{8} \mathrm{H}_{20} \mathrm{~N}\right)\left[\mathrm{PtBr}_{3}\left(\mathrm{C}_{9} \mathrm{H}_{9} \mathrm{NOS}\right)\right]$

744.30

Monoclinic, $P 2_{1} / n$

100

7.7591 (2), 30.4214 (8),

$9.6551(3)$

90, 94.539 (1), 90

2271.87 (11)

4

Mo $K \alpha$

11.55

$0.32 \times 0.30 \times 0.24$

Bruker APEXII CCD

Multi-scan (SADABS;

Bruker, 2014)

$0.052,0.093$

$16951,4418,3675$

Refinement

$R\left[F^{2}>2 \sigma\left(F^{2}\right)\right], w R\left(F^{2}\right), S \quad 0.031,0.081,1.03$

No. of reflections

No. of parameters

$\mathrm{H}$-atom treatment

4418

213

$\mathrm{H}$-atom parameters constrained

$\Delta \rho_{\max }, \Delta \rho_{\min }\left(\mathrm{e} \AA^{-3}\right)$

$2.38,-0.93$
Bruker APEXII CCD

Multi-scan (SADABS;

Bruker, 2014)

$0.056,0.093$

$12741,4650,4377$

0.017

0.626

$0.027,0.066,1.08$

4650

232

$\mathrm{H}$-atom parameters constrained

$1.25,-1.36$
(3)

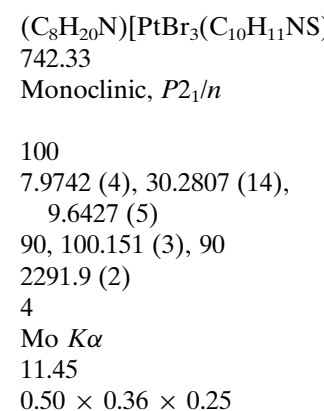

Bruker APEXII CCD

Multi-scan (SADABS;

Bruker, 2014)

$0.003,0.028$

$10729,4692,4120$

0.048

0.627

0.028

0.627

759.28

100

11.83
(4)

$\left(\mathrm{C}_{8} \mathrm{H}_{20} \mathrm{~N}\right)\left[\mathrm{PtBr}_{3}\left(\mathrm{C}_{8} \mathrm{H}_{6} \mathrm{~N}_{2} \mathrm{O}_{2} \mathrm{~S}\right)\right]$

Monoclinic, $P 2_{1} / n$

8.1170 (3), 29.2717 (12), $9.5102(4)$

90, $100.720(1), 90$

$2220.17(15)$

Mo $K \alpha$

$0.32 \times 0.30 \times 0.25$

Bruker APEXII CCD

Multi-scan (SADABS;

Bruker, 2014)

$0.020,0.045$

$15975,4550,4254$

$\begin{array}{ll}0.039,0.106,1.05 & 0.029,0.060,1.18 \\ 4692 & 4550 \\ 266 & 240 \\ \begin{array}{l}\text { H-atom parameters } \\ \text { constrained }\end{array} & \text { H-atom parameters } \\ 1.88,-1.02 & \text { constrained } \\ & 1.25,-1.37\end{array}$

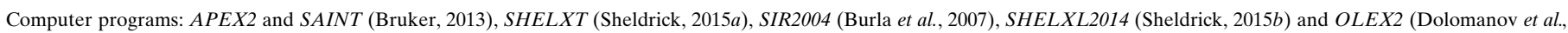
2009).

$7.5(7)^{\circ}$. The $\mathrm{C}-\mathrm{NO}_{2}$ bond length is 1.476 (7) $\AA$, and the $\mathrm{O}-$ $\mathrm{N}-\mathrm{O}$ angle is $117.4(5)^{\circ}$. The $\mathrm{C}-\mathrm{NO}_{2}$ bond length and $\mathrm{O}-$ $\mathrm{N}-\mathrm{O}$ angle in 4 are smaller than those observed in nitrobenzene $\left[\mathrm{C}-\mathrm{NO}_{2}=1.486\right.$ (2) $\AA$ and $\left.\mathrm{O}-\mathrm{N}-\mathrm{O}=123.9(5)^{\circ}\right]$, which suggests higher electron delocalization between the nitro group and the aromatic ring in 4 (Johnson, 2015). The angles at the $\mathrm{S}$ atom in $\mathbf{2 , 3}$ and $\mathbf{4}$ are also near $90^{\circ}$, suggesting the use of pure $p$ orbitals for bonding.

\section{Supramolecular features}

Analysis of the packing diagrams of all of the complexes showed their packings consist of $\left[\mathrm{NEt}_{4}\right]^{+}$cations and $\left[\operatorname{PtBr}_{3}(L)\right]^{-}$anions. The $\left[\mathrm{NEt}_{4}\right]\left[\mathrm{PtBr}_{3}\right.$ (2-Me-benzothiazole $\left.)\right]$ and $\left[\mathrm{NEt}_{4}\right]\left[\mathrm{PtBr}_{3}(6-\mathrm{OMe}-2-\mathrm{Me}-\right.$ benzothiazole $\left.)\right]$ complexes showed partial $\pi$-stacking between the phenyl and the thiazole rings (Fig. 5).

\section{Synthesis and crystallization}

The parent complex $\left[\mathrm{NEt}_{4}\right]_{2}\left[\mathrm{Pt}_{2} \mathrm{Br}_{6}\right]$ was prepared as reported in the literature (Livingstone \& Whitley, 1962). Ligands were purchased from Sigma-Aldrich and were used without further purification.
Acetone solutions of $\left[\mathrm{NEt}_{4}\right]_{2}\left[\mathrm{Pt}_{2} \mathrm{Br}_{6}\right]$ were prepared $(0.075 \mathrm{~g}, 0.068 \mathrm{mmol})$ and the corresponding amount of ligand was added with stirring. For 2-methyl-1,3-benzothiazole (99\%) $18 \mu \mathrm{L}(0.021 \mathrm{~g}, 0.14 \mathrm{mmol})$ were added; for 2-methyl-5-nitro1,3-benzothiazole ( $98 \%)(0.027 \mathrm{~g}, 0.14 \mathrm{mmol})$ were added, and for 2-methyl-6-methoxy-1,3-benzothiazole (97\%) (0.024 g, $0.14 \mathrm{mmol}$ ) were added. The reaction mixtures were stirred without heating until the volume reduced considerably; then the samples were placed in desiccators containing $\mathrm{CaCl}_{2}$ at room temperature to evaporate slowly, leading to the formation of X-ray quality single crystals. For the synthesis with 2,5,6-trimethyl-1,3-benzothiazole $(99 \%)$, the ligand $(0.0227 \mathrm{~g}$, $0.128 \mathrm{mmol}$ ) was added to $20 \mathrm{~mL}$ of an acetone solution $(0.07515 \mathrm{~g}, 0.0677 \mathrm{mmol})$ of $\left[\mathrm{NEt}_{4}\right]_{2}\left[\mathrm{Pt}_{2} \mathrm{Br}_{6}\right]$ with stirring, and a portion of the reaction mixture was slowly evaporated at $277 \mathrm{~K}$ in a small beaker in a secondary container which also contained $\mathrm{CaCl}_{2}$ to form X-ray quality single crystals.

\section{Refinement}

Crystal data, data collection and structure refinement details are summarized in Table $2 . \mathrm{H}$ atoms were positioned in idealized locations: $d(\mathrm{C}-\mathrm{H})=0.95 \AA, U_{\text {iso }}(\mathrm{H})=1.2 U_{\text {eq }}(\mathrm{C})$; $d\left(\mathrm{C}-\mathrm{H}_{2}\right)=0.99 \AA, U_{\text {iso }}(\mathrm{H})=1.2 U_{\mathrm{eq}}(\mathrm{C}) ; d\left(\mathrm{C}-\mathrm{H}_{3}\right)=0.98 \AA$, 
$U_{\text {iso }}(\mathrm{H})=1.5 U_{\text {eq }}(\mathrm{C})$. The $\mathrm{NEt}_{4}$ cation in 3 presented disorder with $0.57 / 0.43$ occupancies.

\section{Acknowledgements}

We thank Ms Lorraine Hernández and Ms Nivia Ruiz-Alago for their help with the synthesis of the platinum compounds. We are grateful to Dr Jorge Rios-Steiner and Mr Daniel J. Vallés-Cádiz for their assistance in the crystallization process. EM thanks the NIH for financial support and JACN acknowledges the financial support of Sloan Program.

\section{References}

Bruker (2013). APEX2 and SAINT. Bruker AXS Inc., Madison, Wisconsin, USA.

Bruker (2014). SADABS. Bruker AXS Inc., Madison, Wisconsin, USA.

Burla, M. C., Caliandro, R., Camalli, M., Carrozzini, B., Cascarano, G. L., De Caro, L., Giacovazzo, C., Polidori, G., Siliqi, D. \& Spagna, R. (2007). J. Appl. Cryst. 40, 609-613.
Dolomanov, O. V., Bourhis, L. J., Gildea, R. J., Howard, J. A. K. \& Puschmann, H. (2009). J. Appl. Cryst. 42, 339-341.

Gomez, G. M., Muir, M. M., Muir, J. A. \& Cox, O. (1988). Acta Cryst. C44, 1554-1557.

Johnson, R. D. III (2015). NIST Computational Chemistry Comparison and Benchmark Database, NIST Standard Reference Database Number 101 Release 17b, September 2015, edited by Russell D. Johnson III. http://cccbdb.NIST.gov/exp2.asp?casno $=98953$ (accessed on November 24, 2015).

Livingstone, S. E. \& Whitley, A. (1962). Aust. J. Chem. 15, 175-180.

Lozano, C. M., Muir, M. M., Tang, X. \& Li, Y. (1994). J. Chem. Cryst. 24, 639-642.

Muir, M. M., Cadiz, M. E. \& Baez, A. (1988a). Inorg. Chim. Acta, 151, 209-213.

Muir, M. M., Gomez, G. M., Cadiz, M. E. \& Muir, J. E. (1990). Inorg. Chim. Acta, 168, 47-57.

Muir, M. M., Gomez, G., Muir, J. A., Cadiz, M. E., Cox, O. \& Barnes, C. L. (1988b). Acta Cryst. C44, 803-806.

Muir, J. A., Gomez, G. M., Muir, M. M., Cox, O. \& Cadiz, M. E. (1987). Acta Cryst. C43, 1258-1261.

Noolvi, M. N., Patel, H. M. \& Kaur, M. (2012). Eur. J. Med. Chem. 54, 447-462.

Sheldrick, G. M. (2015a). Acta Cryst. A71, 3-8.

Sheldrick, G. M. (2015b). Acta Cryst. C71, 3-8. 


\section{supporting information}

Acta Cryst. (2016). E72, 412-416 [doi:10.1107/S2056989016002826]

New platinum(II) complexes with benzothiazole ligands

José A. Carmona-Negrón, Mayra E. Cádiz, Curtis E. Moore, Arnold L. Rheingold and Enrique

Meléndez

Computing details

For all compounds, data collection: APEX2 (Bruker, 2013); cell refinement: SAINT (Bruker, 2013); data reduction: SAINT (Bruker, 2013). Program(s) used to solve structure: SHELXT (Sheldrick, 2015a) for (1), (2), (3); SIR2004 (Burla et al., 2007) for (4). For all compounds, program(s) used to refine structure: SHELXL2014 (Sheldrick, 2015b); molecular graphics: OLEX2 (Dolomanov et al., 2009); software used to prepare material for publication: OLEX2 (Dolomanov et al., 2009).

(1) Tetraethylammonium tribromido(2-methyl-1,3-benzothiazole- $k N$ ) platinate(II)

Crystal data

$\left(\mathrm{C}_{8} \mathrm{H}_{20} \mathrm{~N}\right)\left[\mathrm{PtBr}_{3}\left(\mathrm{C}_{8} \mathrm{H}_{7} \mathrm{NS}\right)\right]$

$M_{r}=714.27$

Orthorhombic, $\mathrm{Pbca}$

$a=12.114(3) \AA$

$b=10.656(3) \AA$

$c=34.043(9) \AA$

$V=4394(2) \AA^{3}$

$Z=8$

$F(000)=2688$

Data collection

Bruker APEXII CCD diffractometer

Radiation source: Micro Focus Rotating Anode, Bruker TXS

Double Bounce Multilayer Mirrors monochromator

Detector resolution: 7.9 pixels $\mathrm{mm}^{-1}$

$\varphi$ and $\omega$ scans

Absorption correction: multi-scan

(SADABS; Bruker, 2014)

Refinement

Refinement on $F^{2}$

Least-squares matrix: full

$R\left[F^{2}>2 \sigma\left(F^{2}\right)\right]=0.031$

$w R\left(F^{2}\right)=0.081$

$S=1.03$

4418 reflections
$D_{\mathrm{x}}=2.159 \mathrm{Mg} \mathrm{m}^{-3}$

Mo $K \alpha$ radiation, $\lambda=0.71073 \AA$

Cell parameters from 5330 reflections

$\theta=2.4-26.3^{\circ}$

$\mu=11.94 \mathrm{~mm}^{-1}$

$T=100 \mathrm{~K}$

Block, bronze

$0.18 \times 0.16 \times 0.12 \mathrm{~mm}$

$T_{\min }=0.052, T_{\max }=0.093$

16951 measured reflections

4418 independent reflections

3675 reflections with $I>2 \sigma(I)$

$R_{\text {int }}=0.047$

$\theta_{\max }=26.3^{\circ}, \theta_{\min }=2.1^{\circ}$

$h=-14 \rightarrow 15$

$k=-13 \rightarrow 10$

$l=-32 \rightarrow 42$

213 parameters

0 restraints

Hydrogen site location: inferred from neighbouring sites

$\mathrm{H}$-atom parameters constrained 
$w=1 /\left[\sigma^{2}\left(F_{\mathrm{o}}^{2}\right)+(0.0352 P)^{2}+9.4131 P\right]$

where $P=\left(F_{\mathrm{o}}^{2}+2 F_{\mathrm{c}}^{2}\right) / 3$

$(\Delta / \sigma)_{\max }=0.003$

$$
\Delta \rho_{\max }=2.38 \text { e } \AA^{-3}
$$

\section{Special details}

Geometry. All e.s.d.'s (except the e.s.d. in the dihedral angle between two l.s. planes) are estimated using the full covariance matrix. The cell e.s.d.'s are taken into account individually in the estimation of e.s.d.'s in distances, angles and torsion angles; correlations between e.s.d.'s in cell parameters are only used when they are defined by crystal symmetry. An approximate (isotropic) treatment of cell e.s.d.'s is used for estimating e.s.d.'s involving 1.s. planes.

Fractional atomic coordinates and isotropic or equivalent isotropic displacement parameters $\left(\hat{A}^{2}\right)$

\begin{tabular}{|c|c|c|c|c|}
\hline & $x$ & $y$ & $z$ & $U_{\text {iso }} * / U_{\text {eq }}$ \\
\hline N2 & $1.0097(4)$ & $0.4493(4)$ & $0.31862(12)$ & $0.0213(9)$ \\
\hline $\mathrm{C} 9$ & $1.1117(4)$ & $0.3738(5)$ & $0.30557(16)$ & $0.0242(12)$ \\
\hline H9A & 1.1049 & 0.2872 & 0.3158 & $0.029 *$ \\
\hline H9B & 1.1781 & 0.4117 & 0.3177 & $0.029 *$ \\
\hline $\mathrm{C} 10$ & $1.1289(4)$ & $0.3677(5)$ & $0.26030(15)$ & $0.0251(12)$ \\
\hline H10A & 1.0624 & 0.3336 & 0.2479 & $0.038^{*}$ \\
\hline H10B & 1.1920 & 0.3134 & 0.2544 & $0.038 *$ \\
\hline $\mathrm{H} 10 \mathrm{C}$ & 1.1431 & 0.4523 & 0.2502 & $0.038^{*}$ \\
\hline C11 & $1.0130(5)$ & $0.5845(5)$ & $0.30315(17)$ & 0.0259 (12) \\
\hline H11A & 1.0120 & 0.5820 & 0.2741 & $0.031 *$ \\
\hline H11B & 0.9452 & 0.6282 & 0.3119 & $0.031^{*}$ \\
\hline C12 & $1.1120(5)$ & $0.6608(5)$ & $0.31618(18)$ & $0.0339(14)$ \\
\hline $\mathrm{H} 12 \mathrm{~A}$ & 1.1104 & 0.7430 & 0.3033 & $0.051 *$ \\
\hline H12B & 1.1799 & 0.6166 & 0.3089 & $0.051 *$ \\
\hline $\mathrm{H} 12 \mathrm{C}$ & 1.1097 & 0.6720 & 0.3447 & $0.051 *$ \\
\hline C13 & $1.0124(5)$ & $0.4436(6)$ & $0.36271(15)$ & $0.0259(12)$ \\
\hline $\mathrm{H} 13 \mathrm{~A}$ & 1.0827 & 0.4809 & 0.3719 & $0.031 *$ \\
\hline H13B & 1.0121 & 0.3543 & 0.3708 & $0.031 *$ \\
\hline C14 & $0.9162(5)$ & $0.5110(5)$ & $0.38363(18)$ & $0.0305(13)$ \\
\hline $\mathrm{H} 14 \mathrm{~A}$ & 0.9172 & 0.6003 & 0.3768 & $0.046^{*}$ \\
\hline H14B & 0.9243 & 0.5017 & 0.4121 & $0.046^{*}$ \\
\hline $\mathrm{H} 14 \mathrm{C}$ & 0.8460 & 0.4739 & 0.3753 & $0.046^{*}$ \\
\hline $\mathrm{C} 15$ & $0.9038(4)$ & $0.3934(5)$ & $0.30181(16)$ & $0.0248(12)$ \\
\hline H15A & 0.8405 & 0.4417 & 0.3122 & $0.030^{*}$ \\
\hline H15B & 0.9048 & 0.4048 & 0.2730 & $0.030^{*}$ \\
\hline C16 & $0.8844(4)$ & $0.2555(5)$ & $0.31049(17)$ & $0.0303(13)$ \\
\hline H16A & 0.9447 & 0.2056 & 0.2993 & $0.045^{*}$ \\
\hline H16B & 0.8142 & 0.2291 & 0.2988 & $0.045^{*}$ \\
\hline $\mathrm{H} 16 \mathrm{C}$ & 0.8819 & 0.2426 & 0.3390 & $0.045^{*}$ \\
\hline Pt1 & $0.47417(2)$ & $0.49411(2)$ & $0.37148(2)$ & $0.01931(8)$ \\
\hline Br1 & $0.31934(5)$ & $0.60895(7)$ & $0.39974(2)$ & 0.04565 (19) \\
\hline $\mathrm{Br} 2$ & $0.39703(4)$ & $0.51437(5)$ & $0.30574(2)$ & $0.02401(13)$ \\
\hline $\mathrm{Br} 3$ & $0.63067(4)$ & $0.37553(6)$ & $0.34693(2)$ & $0.03153(15)$ \\
\hline $\mathrm{S} 1$ & $0.67084(13)$ & $0.48262(14)$ & $0.48520(4)$ & $0.0321(3)$ \\
\hline N1 & $0.5409(4)$ & $0.4702(4)$ & $0.42583(13)$ & $0.0221(10)$ \\
\hline $\mathrm{C} 1$ & $0.6192(4)$ & $0.5394(5)$ & $0.44109(15)$ & $0.0247(12)$ \\
\hline
\end{tabular}




\begin{tabular}{lllll} 
C2 & $0.5158(4)$ & $0.3638(5)$ & $0.44867(15)$ & $0.0246(12)$ \\
C3 & $0.4356(5)$ & $0.2744(5)$ & $0.44047(16)$ & $0.0290(12)$ \\
H3 & 0.3925 & 0.2796 & 0.4172 & $0.035^{*}$ \\
C4 & $0.4200(6)$ & $0.1769(6)$ & $0.46718(16)$ & $0.0351(14)$ \\
H4 & 0.3642 & 0.1162 & 0.4624 & $0.042^{*}$ \\
C5 & $0.4853(5)$ & $0.1671(7)$ & $0.50112(16)$ & $0.0398(16)$ \\
H5 & 0.4740 & 0.0987 & 0.5186 & $0.048^{*}$ \\
C6 & $0.5666(6)$ & $0.2558(6)$ & $0.50970(17)$ & $0.0379(15)$ \\
H6 & 0.6106 & 0.2495 & 0.5327 & $0.045^{*}$ \\
C7 & $0.5807(5)$ & $0.3552(5)$ & $0.48283(16)$ & $0.0315(13)$ \\
C8 & $0.6643(5)$ & $0.6567(5)$ & $0.42301(16)$ & $0.0290(13)$ \\
H8A & 0.7204 & 0.6929 & 0.4404 & $0.044^{*}$ \\
H8B & 0.6043 & 0.7173 & 0.4193 & $0.044^{*}$ \\
H8C & 0.6976 & 0.6368 & 0.3975 & $0.044^{*}$ \\
\hline
\end{tabular}

Atomic displacement parameters $\left(\AA^{2}\right)$

\begin{tabular}{lllllll}
\hline & $U^{11}$ & $U^{22}$ & $U^{33}$ & $U^{12}$ & $U^{13}$ & $U^{23}$ \\
\hline $\mathrm{N} 2$ & $0.022(2)$ & $0.022(2)$ & $0.020(2)$ & $0.0039(19)$ & $0.0002(18)$ & $0.0024(19)$ \\
$\mathrm{C} 9$ & $0.016(3)$ & $0.024(3)$ & $0.032(3)$ & $0.001(2)$ & $0.000(2)$ & $0.000(2)$ \\
$\mathrm{C} 10$ & $0.019(3)$ & $0.025(3)$ & $0.032(3)$ & $0.003(2)$ & $0.004(2)$ & $-0.003(2)$ \\
$\mathrm{C} 11$ & $0.026(3)$ & $0.021(3)$ & $0.031(3)$ & $0.002(2)$ & $0.004(2)$ & $0.006(2)$ \\
$\mathrm{C} 12$ & $0.031(3)$ & $0.027(3)$ & $0.044(4)$ & $0.000(3)$ & $-0.004(3)$ & $0.002(3)$ \\
$\mathrm{C} 13$ & $0.030(3)$ & $0.028(3)$ & $0.020(3)$ & $0.000(3)$ & $-0.001(2)$ & $-0.001(2)$ \\
$\mathrm{C} 14$ & $0.030(3)$ & $0.037(3)$ & $0.025(3)$ & $-0.001(3)$ & $0.000(2)$ & $-0.003(2)$ \\
$\mathrm{C} 15$ & $0.018(3)$ & $0.032(3)$ & $0.025(3)$ & $0.003(2)$ & $-0.005(2)$ & $-0.002(2)$ \\
$\mathrm{C} 16$ & $0.021(3)$ & $0.031(3)$ & $0.039(3)$ & $-0.003(2)$ & $-0.001(2)$ & $0.002(3)$ \\
$\mathrm{P} 1$ & $0.01841(13)$ & $0.02210(12)$ & $0.01742(13)$ & $0.00011(8)$ & $-0.00120(7)$ & $-0.00100(8)$ \\
$\mathrm{Br} 1$ & $0.0399(4)$ & $0.0675(5)$ & $0.0296(3)$ & $0.0248(3)$ & $-0.0059(3)$ & $-0.0135(3)$ \\
$\mathrm{Br} 2$ & $0.0241(3)$ & $0.0255(3)$ & $0.0224(3)$ & $-0.0012(2)$ & $-0.0022(2)$ & $-0.0005(2)$ \\
$\mathrm{Br} 3$ & $0.0235(3)$ & $0.0411(3)$ & $0.0300(3)$ & $0.0027(3)$ & $0.0004(2)$ & $-0.0050(3)$ \\
$\mathrm{S} 1$ & $0.0337(8)$ & $0.0381(8)$ & $0.0245(7)$ & $0.0055(7)$ & $-0.0105(6)$ & $-0.0028(6)$ \\
$\mathrm{N} 1$ & $0.022(2)$ & $0.025(2)$ & $0.019(2)$ & $0.0030(19)$ & $0.0017(18)$ & $0.0000(19)$ \\
$\mathrm{C} 1$ & $0.022(3)$ & $0.031(3)$ & $0.021(3)$ & $0.006(2)$ & $-0.004(2)$ & $-0.009(2)$ \\
$\mathrm{C} 2$ & $0.030(3)$ & $0.026(3)$ & $0.018(3)$ & $0.005(2)$ & $0.001(2)$ & $-0.005(2)$ \\
$\mathrm{C} 3$ & $0.033(3)$ & $0.032(3)$ & $0.021(3)$ & $-0.003(3)$ & $0.004(2)$ & $-0.005(2)$ \\
$\mathrm{C} 4$ & $0.051(4)$ & $0.032(3)$ & $0.022(3)$ & $-0.004(3)$ & $0.006(3)$ & $-0.002(2)$ \\
$\mathrm{C} 5$ & $0.062(4)$ & $0.032(4)$ & $0.026(3)$ & $0.003(3)$ & $0.005(3)$ & $0.003(2)$ \\
$\mathrm{C} 6$ & $0.054(4)$ & $0.035(3)$ & $0.025(3)$ & $0.011(3)$ & $-0.004(3)$ & $0.001(3)$ \\
$\mathrm{C} 7$ & $0.036(3)$ & $0.033(3)$ & $0.025(3)$ & $0.006(3)$ & $0.002(3)$ & $-0.010(2)$ \\
C8 & $0.034(3)$ & $0.032(3)$ & $0.021(3)$ & $-0.001(3)$ & $-0.010(2)$ & $-0.004(2)$ \\
& & & & & & \\
\hline
\end{tabular}

Geometric parameters $\left(A,{ }^{\circ}\right)$

\begin{tabular}{llll}
\hline $\mathrm{N} 2-\mathrm{C} 9$ & $1.540(6)$ & $\mathrm{C} 16-\mathrm{H} 16 \mathrm{~A}$ & 0.9800 \\
$\mathrm{~N} 2-\mathrm{C} 11$ & $1.534(7)$ & $\mathrm{C} 16-\mathrm{H} 16 \mathrm{~B}$ & 0.9800 \\
$\mathrm{~N} 2-\mathrm{C} 13$ & $1.503(7)$ & $\mathrm{C} 16-\mathrm{H} 16 \mathrm{C}$ & 0.9800 \\
$\mathrm{~N} 2-\mathrm{C} 15$ & $1.526(7)$ & $\mathrm{P} 1-\mathrm{Br} 1$ & $2.4375(8)$
\end{tabular}




\begin{tabular}{|c|c|c|c|}
\hline C9-H9A & 0.9900 & $\mathrm{Pt} 1-\mathrm{Br} 2$ & $2.4349(8)$ \\
\hline C9-H9B & 0.9900 & $\mathrm{Pt} 1-\mathrm{Br} 3$ & $2.4268(7)$ \\
\hline $\mathrm{C} 9-\mathrm{C} 10$ & $1.557(7)$ & $\mathrm{Pt} 1-\mathrm{N} 1$ & $2.035(5)$ \\
\hline $\mathrm{C} 10-\mathrm{H} 10 \mathrm{~A}$ & 0.9800 & $\mathrm{~S} 1-\mathrm{C} 1$ & $1.735(6)$ \\
\hline $\mathrm{C} 10-\mathrm{H} 10 \mathrm{~B}$ & 0.9800 & $\mathrm{~S} 1-\mathrm{C} 7$ & $1.744(6)$ \\
\hline $\mathrm{C} 10-\mathrm{H} 10 \mathrm{C}$ & 0.9800 & $\mathrm{~N} 1-\mathrm{C} 1$ & $1.309(7)$ \\
\hline $\mathrm{C} 11-\mathrm{H} 11 \mathrm{~A}$ & 0.9900 & $\mathrm{~N} 1-\mathrm{C} 2$ & $1.408(7)$ \\
\hline $\mathrm{C} 11-\mathrm{H} 11 \mathrm{~B}$ & 0.9900 & $\mathrm{C} 1-\mathrm{C} 8$ & $1.497(8)$ \\
\hline $\mathrm{C} 11-\mathrm{C} 12$ & $1.515(8)$ & $\mathrm{C} 2-\mathrm{C} 3$ & $1.389(8)$ \\
\hline $\mathrm{C} 12-\mathrm{H} 12 \mathrm{~A}$ & 0.9800 & $\mathrm{C} 2-\mathrm{C} 7$ & $1.407(8)$ \\
\hline $\mathrm{C} 12-\mathrm{H} 12 \mathrm{~B}$ & 0.9800 & $\mathrm{C} 3-\mathrm{H} 3$ & 0.9500 \\
\hline $\mathrm{C} 12-\mathrm{H} 12 \mathrm{C}$ & 0.9800 & $\mathrm{C} 3-\mathrm{C} 4$ & $1.393(8)$ \\
\hline $\mathrm{C} 13-\mathrm{H} 13 \mathrm{~A}$ & 0.9900 & $\mathrm{C} 4-\mathrm{H} 4$ & 0.9500 \\
\hline C13-H13B & 0.9900 & $\mathrm{C} 4-\mathrm{C} 5$ & $1.405(8)$ \\
\hline $\mathrm{C} 13-\mathrm{C} 14$ & $1.543(8)$ & $\mathrm{C} 5-\mathrm{H} 5$ & 0.9500 \\
\hline $\mathrm{C} 14-\mathrm{H} 14 \mathrm{~A}$ & 0.9800 & $\mathrm{C} 5-\mathrm{C} 6$ & $1.396(9)$ \\
\hline C14-H14B & 0.9800 & $\mathrm{C} 6-\mathrm{H} 6$ & 0.9500 \\
\hline $\mathrm{C} 14-\mathrm{H} 14 \mathrm{C}$ & 0.9800 & $\mathrm{C} 6-\mathrm{C} 7$ & $1.410(8)$ \\
\hline $\mathrm{C} 15-\mathrm{H} 15 \mathrm{~A}$ & 0.9900 & $\mathrm{C} 8-\mathrm{H} 8 \mathrm{~A}$ & 0.9800 \\
\hline C15-H15B & 0.9900 & $\mathrm{C} 8-\mathrm{H} 8 \mathrm{~B}$ & 0.9800 \\
\hline $\mathrm{C} 15-\mathrm{C} 16$ & $1.517(8)$ & $\mathrm{C} 8-\mathrm{H} 8 \mathrm{C}$ & 0.9800 \\
\hline $\mathrm{C} 11-\mathrm{N} 2-\mathrm{C} 9$ & $111.8(4)$ & $\mathrm{C} 16-\mathrm{C} 15-\mathrm{H} 15 \mathrm{~A}$ & 108.3 \\
\hline $\mathrm{C} 13-\mathrm{N} 2-\mathrm{C} 9$ & $104.5(4)$ & $\mathrm{C} 16-\mathrm{C} 15-\mathrm{H} 15 \mathrm{~B}$ & 108.3 \\
\hline $\mathrm{C} 13-\mathrm{N} 2-\mathrm{C} 11$ & $112.4(4)$ & $\mathrm{C} 15-\mathrm{C} 16-\mathrm{H} 16 \mathrm{~A}$ & 109.5 \\
\hline $\mathrm{C} 13-\mathrm{N} 2-\mathrm{C} 15$ & $112.1(4)$ & $\mathrm{C} 15-\mathrm{C} 16-\mathrm{H} 16 \mathrm{~B}$ & 109.5 \\
\hline $\mathrm{C} 15-\mathrm{N} 2-\mathrm{C} 9$ & $111.2(4)$ & $\mathrm{C} 15-\mathrm{C} 16-\mathrm{H} 16 \mathrm{C}$ & 109.5 \\
\hline $\mathrm{C} 15-\mathrm{N} 2-\mathrm{C} 11$ & $105.1(4)$ & $\mathrm{H} 16 \mathrm{~A}-\mathrm{C} 16-\mathrm{H} 16 \mathrm{~B}$ & 109.5 \\
\hline $\mathrm{N} 2-\mathrm{C} 9-\mathrm{H} 9 \mathrm{~A}$ & 108.6 & $\mathrm{H} 16 \mathrm{~A}-\mathrm{C} 16-\mathrm{H} 16 \mathrm{C}$ & 109.5 \\
\hline $\mathrm{N} 2-\mathrm{C} 9-\mathrm{H} 9 \mathrm{~B}$ & 108.6 & $\mathrm{H} 16 \mathrm{~B}-\mathrm{C} 16-\mathrm{H} 16 \mathrm{C}$ & 109.5 \\
\hline $\mathrm{N} 2-\mathrm{C} 9-\mathrm{C} 10$ & $114.5(4)$ & $\mathrm{Br} 2-\mathrm{Pt} 1-\mathrm{Br} 1$ & $91.31(2)$ \\
\hline $\mathrm{H} 9 \mathrm{~A}-\mathrm{C} 9-\mathrm{H} 9 \mathrm{~B}$ & 107.6 & $\mathrm{Br} 3-\mathrm{Pt} 1-\mathrm{Br} 1$ & $176.85(2)$ \\
\hline $\mathrm{C} 10-\mathrm{C} 9-\mathrm{H} 9 \mathrm{~A}$ & 108.6 & $\mathrm{Br} 3-\mathrm{Pt} 1-\mathrm{Br} 2$ & $91.69(2)$ \\
\hline $\mathrm{C} 10-\mathrm{C} 9-\mathrm{H} 9 \mathrm{~B}$ & 108.6 & $\mathrm{~N} 1-\mathrm{Pt} 1-\mathrm{Br} 1$ & $90.56(12)$ \\
\hline $\mathrm{C} 9-\mathrm{C} 10-\mathrm{H} 10 \mathrm{~A}$ & 109.5 & $\mathrm{~N} 1-\mathrm{Pt} 1-\mathrm{Br} 2$ & $177.68(13)$ \\
\hline $\mathrm{C} 9-\mathrm{C} 10-\mathrm{H} 10 \mathrm{~B}$ & 109.5 & $\mathrm{~N} 1-\mathrm{Pt} 1-\mathrm{Br} 3$ & $86.41(12)$ \\
\hline $\mathrm{C} 9-\mathrm{C} 10-\mathrm{H} 10 \mathrm{C}$ & 109.5 & $\mathrm{C} 1-\mathrm{S} 1-\mathrm{C} 7$ & $90.3(3)$ \\
\hline $\mathrm{H} 10 \mathrm{~A}-\mathrm{C} 10-\mathrm{H} 10 \mathrm{~B}$ & 109.5 & $\mathrm{C} 1-\mathrm{N} 1-\mathrm{Pt} 1$ & $125.3(4)$ \\
\hline $\mathrm{H} 10 \mathrm{~A}-\mathrm{C} 10-\mathrm{H} 10 \mathrm{C}$ & 109.5 & $\mathrm{C} 1-\mathrm{N} 1-\mathrm{C} 2$ & $113.0(5)$ \\
\hline $\mathrm{H} 10 \mathrm{~B}-\mathrm{C} 10-\mathrm{H} 10 \mathrm{C}$ & 109.5 & $\mathrm{C} 2-\mathrm{N} 1-\mathrm{Pt} 1$ & $121.2(4)$ \\
\hline N2-C11-H11A & 108.5 & $\mathrm{~N} 1-\mathrm{C} 1-\mathrm{S} 1$ & $114.1(4)$ \\
\hline $\mathrm{N} 2-\mathrm{C} 11-\mathrm{H} 11 \mathrm{~B}$ & 108.5 & $\mathrm{~N} 1-\mathrm{C} 1-\mathrm{C} 8$ & $124.9(5)$ \\
\hline $\mathrm{H} 11 \mathrm{~A}-\mathrm{C} 11-\mathrm{H} 11 \mathrm{~B}$ & 107.5 & $\mathrm{C} 8-\mathrm{C} 1-\mathrm{S} 1$ & $121.0(4)$ \\
\hline $\mathrm{C} 12-\mathrm{C} 11-\mathrm{N} 2$ & $115.1(5)$ & $\mathrm{C} 3-\mathrm{C} 2-\mathrm{N} 1$ & $126.3(5)$ \\
\hline $\mathrm{C} 12-\mathrm{C} 11-\mathrm{H} 11 \mathrm{~A}$ & 108.5 & $\mathrm{C} 3-\mathrm{C} 2-\mathrm{C} 7$ & $120.8(5)$ \\
\hline $\mathrm{C} 12-\mathrm{C} 11-\mathrm{H} 11 \mathrm{~B}$ & 108.5 & $\mathrm{C} 7-\mathrm{C} 2-\mathrm{N} 1$ & $112.8(5)$ \\
\hline $\mathrm{C} 11-\mathrm{C} 12-\mathrm{H} 12 \mathrm{~A}$ & 109.5 & $\mathrm{C} 2-\mathrm{C} 3-\mathrm{H} 3$ & 120.8 \\
\hline $\mathrm{C} 11-\mathrm{C} 12-\mathrm{H} 12 \mathrm{~B}$ & 109.5 & $\mathrm{C} 2-\mathrm{C} 3-\mathrm{C} 4$ & $118.4(5)$ \\
\hline
\end{tabular}




$\begin{array}{llll}\mathrm{C} 11-\mathrm{C} 12-\mathrm{H} 12 \mathrm{C} & 109.5 & \mathrm{C} 4-\mathrm{C} 3-\mathrm{H} 3 & 120.8 \\ \mathrm{H} 12 \mathrm{~A}-\mathrm{C} 12-\mathrm{H} 12 \mathrm{~B} & 109.5 & \mathrm{C} 3-\mathrm{C} 4-\mathrm{H} 4 & 119.5 \\ \mathrm{H} 12 \mathrm{~A}-\mathrm{C} 12-\mathrm{H} 12 \mathrm{C} & 109.5 & \mathrm{C} 3-\mathrm{C} 4-\mathrm{C} 5 & 121.0(6) \\ \mathrm{H} 12 \mathrm{~B}-\mathrm{C} 12-\mathrm{H} 12 \mathrm{C} & 109.5 & \mathrm{C} 5-\mathrm{C} 4-\mathrm{H} 4 & 119.5 \\ \mathrm{~N} 2-\mathrm{C} 13-\mathrm{H} 13 \mathrm{~A} & 108.5 & \mathrm{C} 4-\mathrm{C} 5-\mathrm{H} 5 & 119.4 \\ \mathrm{~N} 2-\mathrm{C} 13-\mathrm{H} 13 \mathrm{~B} & 108.5 & \mathrm{C} 6-\mathrm{C} 5-\mathrm{C} 4 & 121.3(6) \\ \mathrm{N} 2-\mathrm{C} 13-\mathrm{C} 14 & 115.2(5) & \mathrm{C} 6-\mathrm{C} 5-\mathrm{H} 5 & 119.4 \\ \mathrm{H} 13 \mathrm{~A}-\mathrm{C} 13-\mathrm{H} 13 \mathrm{~B} & 107.5 & \mathrm{C} 5-\mathrm{C} 6-\mathrm{H} 6 & 121.4 \\ \mathrm{C} 14-\mathrm{C} 13-\mathrm{H} 13 \mathrm{~A} & 108.5 & \mathrm{C} 5-\mathrm{C} 6-\mathrm{C} 7 & 121.4 \\ \mathrm{C} 14-\mathrm{C} 13-\mathrm{H} 13 \mathrm{~B} & 108.5 & \mathrm{C} 7-\mathrm{C} 6-\mathrm{H} 6 & 109.7(4) \\ \mathrm{C} 13-\mathrm{C} 14-\mathrm{H} 14 \mathrm{~A} & 109.5 & \mathrm{C} 2-\mathrm{C} 7-\mathrm{S} 1 & 121.2(6) \\ \mathrm{C} 13-\mathrm{C} 14-\mathrm{H} 14 \mathrm{~B} & 109.5 & \mathrm{C} 2-\mathrm{C} 7-\mathrm{C} 6 & 129.1(5) \\ \mathrm{C} 13-\mathrm{C} 14-\mathrm{H} 14 \mathrm{C} & 109.5 & \mathrm{C} 6-\mathrm{C} 7-\mathrm{S} 1 & 109.5 \\ \mathrm{H} 14 \mathrm{~A}-\mathrm{C} 14-\mathrm{H} 14 \mathrm{~B} & 109.5 & \mathrm{C} 1-\mathrm{C} 8-\mathrm{H} 8 \mathrm{~A} & 109.5 \\ \mathrm{H} 14 \mathrm{~A}-\mathrm{C} 14-\mathrm{H} 14 \mathrm{C} & 109.5 & \mathrm{C} 1-\mathrm{C} 8-\mathrm{H} 8 \mathrm{~B} & 109.5 \\ \mathrm{H} 14 \mathrm{~B}-\mathrm{C} 14-\mathrm{H} 14 \mathrm{C} & 109.5 & \mathrm{C} 1-\mathrm{C} 8-\mathrm{H} 8 \mathrm{C} & 109.5 \\ \text { N2-C15-H15A } & 108.3 & \mathrm{H} 8 \mathrm{~A}-\mathrm{C} 8-\mathrm{H} 8 \mathrm{~B} & 109.5 \\ \text { N2-C15-H15B } & 108.3 & \mathrm{H} 8 \mathrm{~A}-\mathrm{C} 8-\mathrm{H} 8 \mathrm{C} & \\ \mathrm{H} 15 \mathrm{~A}-\mathrm{C} 15-\mathrm{H} 15 \mathrm{~B} & 107.4 & \mathrm{H} 8 \mathrm{~B}-\mathrm{C} 8-\mathrm{H} 8 \mathrm{C} & \\ \text { C16-C15-N2 } & 115.8(4) & & \end{array}$

(2) Tetraethylammonium tribromido(6-methoxy-2-methyl-1,3-benzothiazole- $\kappa \mathrm{N}$ ) platinate(II)

\section{Crystal data}

$\left(\mathrm{C}_{8} \mathrm{H}_{20} \mathrm{~N}\right)\left[\mathrm{PtBr}_{3}\left(\mathrm{C}_{9} \mathrm{H}_{9} \mathrm{NOS}\right)\right]$

$M_{r}=744.30$

Monoclinic, $P 2_{1} / n$

$a=7.7591(2) \AA$

$b=30.4214(8) \AA$

$c=9.6551(3) \AA$

$\beta=94.539(1)^{\circ}$

$V=2271.87(11) \AA^{3}$

$Z=4$

\section{Data collection}

Bruker APEXII CCD diffractometer

Radiation source: Micro Focus Rotating Anode, Bruker TXS

Double Bounce Multilayer Mirrors monochromator

Detector resolution: 7.9 pixels $\mathrm{mm}^{-1}$

$\varphi$ and $\omega$ scans

Absorption correction: multi-scan

(SADABS; Bruker, 2014)

\section{Refinement}

Refinement on $F^{2}$

Least-squares matrix: full

$R\left[F^{2}>2 \sigma\left(F^{2}\right)\right]=0.027$

$w R\left(F^{2}\right)=0.066$

$S=1.08$
$F(000)=1408$

$D_{\mathrm{x}}=2.176 \mathrm{Mg} \mathrm{m}^{-3}$

Mo $K \alpha$ radiation, $\lambda=0.71073 \AA$

Cell parameters from 7838 reflections

$\theta=2.2-26.4^{\circ}$

$\mu=11.55 \mathrm{~mm}^{-1}$

$T=100 \mathrm{~K}$

Block, bronze

$0.32 \times 0.3 \times 0.24 \mathrm{~mm}$

$T_{\min }=0.056, T_{\max }=0.093$

12741 measured reflections

4650 independent reflections

4377 reflections with $I>2 \sigma(I)$

$R_{\text {int }}=0.017$

$\theta_{\text {max }}=26.4^{\circ}, \theta_{\min }=2.2^{\circ}$

$h=-9 \rightarrow 9$

$k=-32 \rightarrow 38$

$l=-12 \rightarrow 7$

4650 reflections

232 parameters

0 restraints

Hydrogen site location: inferred from neighbouring sites 
H-atom parameters constrained

$w=1 /\left[\sigma^{2}\left(F_{\mathrm{o}}^{2}\right)+(0.0227 P)^{2}+15.6321 P\right]$

where $P=\left(F_{\mathrm{o}}^{2}+2 F_{\mathrm{c}}^{2}\right) / 3$

$$
\begin{aligned}
& (\Delta / \sigma)_{\max }=0.001 \\
& \Delta \rho_{\max }=1.25 \mathrm{e} \AA^{-3} \\
& \Delta \rho_{\min }=-1.36 \mathrm{e} \AA^{-3}
\end{aligned}
$$

Special details

Geometry. All e.s.d.'s (except the e.s.d. in the dihedral angle between two l.s. planes) are estimated using the full covariance matrix. The cell e.s.d.'s are taken into account individually in the estimation of e.s.d.'s in distances, angles and torsion angles; correlations between e.s.d.'s in cell parameters are only used when they are defined by crystal symmetry.

\begin{tabular}{|c|c|c|c|c|}
\hline & $x$ & $y$ & $z$ & $U_{\text {iso }} * / U_{\text {eq }}$ \\
\hline $\mathrm{N} 2$ & $0.4744(6)$ & $0.68841(14)$ & $1.0072(4)$ & $0.0224(9)$ \\
\hline $\mathrm{C} 10$ & $0.4365(10)$ & $0.6636(2)$ & $0.8727(7)$ & $0.0442(16)$ \\
\hline $\mathrm{H} 10 \mathrm{~A}$ & 0.3248 & 0.6744 & 0.8289 & $0.053 *$ \\
\hline H10B & 0.5266 & 0.6715 & 0.8099 & $0.053^{*}$ \\
\hline C11 & $0.4277(12)$ & $0.6153(2)$ & $0.8795(10)$ & $0.065(3)$ \\
\hline H11A & 0.5326 & 0.6039 & 0.9305 & $0.097 *$ \\
\hline H11B & 0.4183 & 0.6032 & 0.7851 & $0.097 *$ \\
\hline $\mathrm{H} 11 \mathrm{C}$ & 0.3263 & 0.6065 & 0.9274 & $0.097^{*}$ \\
\hline $\mathrm{C} 12$ & $0.6550(9)$ & $0.6759(3)$ & $1.0680(8)$ & $0.0529(19)$ \\
\hline $\mathrm{H} 12 \mathrm{~A}$ & 0.6514 & 0.6451 & 1.1004 & $0.064 *$ \\
\hline H12B & 0.7336 & 0.6769 & 0.9921 & $0.064^{*}$ \\
\hline C13 & $0.7308(9)$ & 0.7028 & $1.1828(7)$ & $0.061(2)$ \\
\hline H13A & 0.6425 & 0.7094 & 1.2470 & $0.092 *$ \\
\hline H13B & 0.7743 & 0.7304 & 1.1462 & $0.092 *$ \\
\hline $\mathrm{H} 13 \mathrm{C}$ & 0.8264 & 0.6868 & 1.2322 & $0.092 *$ \\
\hline C14 & $0.3484(8)$ & $0.6760(2)$ & $1.1136(7)$ & $0.0403(15)$ \\
\hline $\mathrm{H} 14 \mathrm{~A}$ & 0.3582 & 0.6440 & 1.1319 & $0.048^{*}$ \\
\hline H14B & 0.3825 & 0.6915 & 1.2018 & $0.048^{*}$ \\
\hline $\mathrm{C} 15$ & $0.1616(7)$ & $0.6868(2)$ & $1.0702(6)$ & $0.0338(13)$ \\
\hline $\mathrm{H} 15 \mathrm{~A}$ & 0.0883 & 0.6767 & 1.1421 & $0.051 *$ \\
\hline H15B & 0.1267 & 0.6719 & 0.9824 & $0.051^{*}$ \\
\hline $\mathrm{H} 15 \mathrm{C}$ & 0.1488 & 0.7186 & 1.0581 & $0.051^{*}$ \\
\hline C16 & $0.4651(9)$ & $0.7366(2)$ & $0.9800(8)$ & $0.0426(15)$ \\
\hline H16A & 0.4764 & 0.7522 & 1.0703 & $0.051 *$ \\
\hline H16B & 0.3493 & 0.7435 & 0.9349 & $0.051 *$ \\
\hline C17 & $0.6004(9)$ & $0.7545(2)$ & $0.8898(7)$ & $0.0425(15)$ \\
\hline H17A & 0.7160 & 0.7485 & 0.9340 & $0.064^{*}$ \\
\hline H17B & 0.5849 & 0.7864 & 0.8787 & $0.064 *$ \\
\hline $\mathrm{H} 17 \mathrm{C}$ & 0.5876 & 0.7404 & 0.7984 & $0.064^{*}$ \\
\hline Pt1 & $0.73847(2)$ & 0.62959 (2) & $0.53521(2)$ & $0.01668(6)$ \\
\hline Br1 & $0.90363(7)$ & $0.66953(2)$ & $0.71969(5)$ & $0.02744(12)$ \\
\hline $\mathrm{Br} 2$ & $0.52471(7)$ & $0.68778(2)$ & $0.51179(5)$ & $0.02766(12)$ \\
\hline $\mathrm{Br} 3$ & $0.58868(7)$ & $0.58627(2)$ & $0.35144(6)$ & $0.02867(12)$ \\
\hline $\mathrm{S} 1$ & $1.20643(16)$ & $0.54060(4)$ & $0.58464(13)$ & $0.0233(3)$ \\
\hline $\mathrm{O} 1$ & $0.8812(6)$ & $0.43871(13)$ & $0.9169(4)$ & $0.0337(9)$ \\
\hline N1 & $0.9166(5)$ & $0.58111(13)$ & $0.5642(4)$ & $0.0184(8)$ \\
\hline
\end{tabular}
An approximate (isotropic) treatment of cell e.s.d.'s is used for estimating e.s.d.'s involving 1.s. planes.

Fractional atomic coordinates and isotropic or equivalent isotropic displacement parameters $\left(\hat{A}^{2}\right)$ 


$\begin{array}{lllll}\text { C1 } & 1.0717(6) & 0.58239(17) & 0.5202(5) & 0.0213(10) \\ \text { C2 } & 0.8935(6) & 0.54544(15) & 0.6519(5) & 0.0191(10) \\ \text { C3 } & 0.7394(7) & 0.53338(17) & 0.7090(5) & 0.0222(10) \\ \text { H3 } & 0.6361 & 0.5497 & 0.6889 & 0.027^{*} \\ \text { C4 } & 0.7421(7) & 0.49725(17) & 0.7951(5) & 0.0255(11) \\ \text { H4 } & 0.6390 & 0.4885 & 0.8344 & 0.031^{*} \\ \text { C5 } & 0.8937(8) & 0.47324(17) & 0.8256(5) & 0.0262(11) \\ \text { C6 } & 1.0457(7) & 0.48345(17) & 0.7664(5) & 0.0250(11) \\ \text { H6 } & 1.1476 & 0.4664 & 0.7841 & 0.030^{*} \\ \text { C7 } & 1.0411(6) & 0.52035(16) & 0.6788(5) & 0.0204(10) \\ \text { C8 } & 1.1316(7) & 0.61649(18) & 0.4252(6) & 0.0277(11) \\ \text { H8A } & 1.1838 & 0.6409 & 0.4799 & 0.042^{*} \\ \text { H8B } & 1.0329 & 0.6273 & 0.3652 & 0.042^{*} \\ \text { H8C } & 1.2175 & 0.6038 & 0.3678 & 0.042^{*} \\ \text { C9 } & 1.0403(9) & 0.4191(2) & 0.9692(7) & 0.0393(15) \\ \text { H9A } & 1.0908 & 0.4030 & 0.8943 & 0.059^{*} \\ \text { H9B } & 1.0187 & 0.3987 & 1.0445 & 0.059^{*} \\ \text { H9C } & 1.1206 & 0.4420 & 1.0046 & 0.059^{*} \\ \end{array}$

Atomic displacement parameters $\left(\AA^{2}\right)$

\begin{tabular}{|c|c|c|c|c|c|c|}
\hline & $U^{11}$ & $U^{22}$ & $U^{\beta 3}$ & $U^{12}$ & $U^{13}$ & $U^{23}$ \\
\hline $\mathrm{N} 2$ & $0.024(2)$ & $0.024(2)$ & $0.019(2)$ & $0.0044(18)$ & $0.0044(17)$ & $0.0013(17)$ \\
\hline $\mathrm{C} 10$ & $0.056(4)$ & $0.044(4)$ & 0.035 & $-0.010(3)$ & $0.015(3)$ & $-0.011(3)$ \\
\hline C11 & $0.076(6)$ & $0.040(4)$ & $0.086(6)$ & -0.017 (4) & $0.048(5)$ & $-0.026(4)$ \\
\hline $\mathrm{C} 12$ & $0.033(4)$ & $0.079(6)$ & $0.047(4)$ & $0.012(4)$ & 0.008 (3) & $0.020(4)$ \\
\hline $\mathrm{C} 13$ & $0.026(3)$ & $0.130(8)$ & $0.027(3)$ & -0.007 (4) & $-0.004(3)$ & $0.005(4)$ \\
\hline C14 & $0.037(3)$ & $0.051(4)$ & $0.033(3)$ & $0.003(3)$ & 0.009 (3) & $0.003(3)$ \\
\hline $\mathrm{C} 15$ & $0.026(3)$ & $0.042(4)$ & $0.034(3)$ & $0.000(2)$ & $0.003(2)$ & -0.008 \\
\hline $\mathrm{C} 16$ & $0.047(4)$ & $0.029(3)$ & $0.052(4)$ & -0.001 & $0.003(3)$ & $-0.004(3)$ \\
\hline $\mathrm{C} 17$ & $0.045(4)$ & $0.034(3)$ & $0.049(4)$ & -0.007 (3) & $0.007(3)$ & $0.005(3)$ \\
\hline Pt1 & $0.01677(10)$ & $0.01604(10)$ & $0.01721(10)$ & $0.00084(7)$ & $0.00130(7)$ & $0.00005(7)$ \\
\hline Br1 & $0.0303(3)$ & $0.0270(3)$ & $0.0244(2)$ & $-0.0012(2)$ & $-0.0021(2)$ & $-0.0027(2)$ \\
\hline $\mathrm{Br} 2$ & $0.0295(3)$ & $0.0267(3)$ & $0.0266(3)$ & $0.0050(2)$ & $0.0015(2)$ & $-0.0004(2)$ \\
\hline $\mathrm{Br} 3$ & $0.0262(3)$ & $0.0286(3)$ & $0.0303(3)$ & 0.0029 (2) & $-0.0035(2)$ & $-0.0062(2)$ \\
\hline S1 & $0.0177(6)$ & $0.0245(6)$ & $0.0275(6)$ & $0.0036(5)$ & $0.0004(5)$ & $-0.0025(5)$ \\
\hline $\mathrm{O} 1$ & 0.047 (2) & $0.022(2)$ & $0.032(2)$ & $0.0026(18)$ & $0.0023(18)$ & $0.0078(16)$ \\
\hline N1 & $0.019(2)$ & 0.018 (2) & $0.0171(19)$ & $-0.0008(16)$ & $-0.0012(16)$ & $-0.0010(16)$ \\
\hline $\mathrm{C} 1$ & $0.018(2)$ & $0.022(3)$ & $0.023(2)$ & 0.0007 (19) & $-0.0009(19)$ & $-0.003(2)$ \\
\hline $\mathrm{C} 2$ & $0.024(2)$ & $0.013(2)$ & $0.020(2)$ & 0.0017 (19) & $0.0000(19)$ & $-0.0010(18)$ \\
\hline $\mathrm{C} 3$ & $0.021(2)$ & 0.020 & $0.026(2)$ & $0.001(2)$ & $0.004(2)$ & $-0.002(2)$ \\
\hline $\mathrm{C} 4$ & 0.028 & $0.024(3)$ & 0.025 & $-0.001(2)$ & $0.007(2)$ & $0.001(2)$ \\
\hline $\mathrm{C} 5$ & $0.041(3)$ & $0.016(2)$ & $0.021(2)$ & $0.000(2)$ & $0.000(2)$ & $-0.0010(19)$ \\
\hline C6 & $0.033(3)$ & $0.017(2)$ & $0.025(2)$ & $0.005(2)$ & $-0.004(2)$ & $-0.002(2)$ \\
\hline $\mathrm{C} 7$ & $0.021(2)$ & $0.018(2)$ & $0.022(2)$ & $0.0006(19)$ & $-0.0022(19)$ & $-0.0056(19)$ \\
\hline $\mathrm{C} 8$ & $0.024(3)$ & $0.027(3)$ & 0.033 & $-0.001(2)$ & $0.008(2)$ & $0.000(2)$ \\
\hline C9 & $0.053(4)$ & 0.025 & $0.037(3)$ & $-0.001(3)$ & $-0.011(3)$ & 0.007 (2) \\
\hline
\end{tabular}


Geometric parameters $\left(\AA,{ }^{\circ}\right)$

\begin{tabular}{|c|c|c|c|}
\hline $\mathrm{N} 2-\mathrm{C} 10$ & $1.511(7)$ & $\mathrm{C} 17-\mathrm{H} 17 \mathrm{C}$ & 0.9800 \\
\hline $\mathrm{N} 2-\mathrm{C} 12$ & $1.523(8)$ & $\mathrm{Pt} 1-\mathrm{Br} 1$ & $2.4352(5)$ \\
\hline $\mathrm{N} 2-\mathrm{C} 14$ & $1.521(7)$ & $\mathrm{Pt} 1-\mathrm{Br} 2$ & $2.4241(6)$ \\
\hline $\mathrm{N} 2-\mathrm{C} 16$ & $1.491(8)$ & $\mathrm{Pt} 1-\mathrm{Br} 3$ & $2.4309(5)$ \\
\hline $\mathrm{C} 10-\mathrm{H} 10 \mathrm{~A}$ & 0.9900 & Pt1-N1 & $2.025(4)$ \\
\hline $\mathrm{C} 10-\mathrm{H} 10 \mathrm{~B}$ & 0.9900 & $\mathrm{~S} 1-\mathrm{C} 1$ & $1.730(5)$ \\
\hline $\mathrm{C} 10-\mathrm{C} 11$ & $1.474(10)$ & $\mathrm{S} 1-\mathrm{C} 7$ & $1.743(5)$ \\
\hline $\mathrm{C} 11-\mathrm{H} 11 \mathrm{~A}$ & 0.9800 & $\mathrm{O} 1-\mathrm{C} 5$ & $1.379(6)$ \\
\hline $\mathrm{C} 11-\mathrm{H} 11 \mathrm{~B}$ & 0.9800 & $\mathrm{O} 1-\mathrm{C} 9$ & $1.427(7)$ \\
\hline $\mathrm{C} 11-\mathrm{H} 11 \mathrm{C}$ & 0.9800 & $\mathrm{~N} 1-\mathrm{C} 1$ & $1.309(6)$ \\
\hline $\mathrm{C} 12-\mathrm{H} 12 \mathrm{~A}$ & 0.9900 & $\mathrm{~N} 1-\mathrm{C} 2$ & $1.396(6)$ \\
\hline C12-H12B & 0.9900 & $\mathrm{C} 1-\mathrm{C} 8$ & $1.483(7)$ \\
\hline $\mathrm{C} 12-\mathrm{C} 13$ & $1.464(11)$ & $\mathrm{C} 2-\mathrm{C} 3$ & $1.405(7)$ \\
\hline $\mathrm{C} 13-\mathrm{H} 13 \mathrm{~A}$ & 0.9800 & $\mathrm{C} 2-\mathrm{C} 7$ & $1.383(7)$ \\
\hline C13-H13B & 0.9800 & $\mathrm{C} 3-\mathrm{H} 3$ & 0.9500 \\
\hline $\mathrm{C} 13-\mathrm{H} 13 \mathrm{C}$ & 0.9800 & $\mathrm{C} 3-\mathrm{C} 4$ & $1.377(7)$ \\
\hline $\mathrm{C} 14-\mathrm{H} 14 \mathrm{~A}$ & 0.9900 & $\mathrm{C} 4-\mathrm{H} 4$ & 0.9500 \\
\hline C14-H14B & 0.9900 & $\mathrm{C} 4-\mathrm{C} 5$ & $1.396(8)$ \\
\hline $\mathrm{C} 14-\mathrm{C} 15$ & $1.513(8)$ & $\mathrm{C} 5-\mathrm{C} 6$ & $1.386(8)$ \\
\hline C15-H15A & 0.9800 & C6- 6 6 & 0.9500 \\
\hline C15-H15B & 0.9800 & $\mathrm{C} 6-\mathrm{C} 7$ & $1.404(7)$ \\
\hline $\mathrm{C} 15-\mathrm{H} 15 \mathrm{C}$ & 0.9800 & $\mathrm{C} 8-\mathrm{H} 8 \mathrm{~A}$ & 0.9800 \\
\hline C16-H16A & 0.9900 & $\mathrm{C} 8-\mathrm{H} 8 \mathrm{~B}$ & 0.9800 \\
\hline C16-H16B & 0.9900 & $\mathrm{C} 8-\mathrm{H} 8 \mathrm{C}$ & 0.9800 \\
\hline $\mathrm{C} 16-\mathrm{C} 17$ & $1.517(9)$ & C9-H9A & 0.9800 \\
\hline C17-H17A & 0.9800 & C9-H9B & 0.9800 \\
\hline C17-H17B & 0.9800 & $\mathrm{C} 9-\mathrm{H} 9 \mathrm{C}$ & 0.9800 \\
\hline $\mathrm{C} 10-\mathrm{N} 2-\mathrm{C} 12$ & $108.5(5)$ & $\mathrm{C} 16-\mathrm{C} 17-\mathrm{H} 17 \mathrm{~B}$ & 109.5 \\
\hline $\mathrm{C} 10-\mathrm{N} 2-\mathrm{C} 14$ & $111.4(5)$ & $\mathrm{C} 16-\mathrm{C} 17-\mathrm{H} 17 \mathrm{C}$ & 109.5 \\
\hline $\mathrm{C} 14-\mathrm{N} 2-\mathrm{C} 12$ & $107.4(4)$ & $\mathrm{H} 17 \mathrm{~A}-\mathrm{C} 17-\mathrm{H} 17 \mathrm{~B}$ & 109.5 \\
\hline $\mathrm{C} 16-\mathrm{N} 2-\mathrm{C} 10$ & $109.6(5)$ & $\mathrm{H} 17 \mathrm{~A}-\mathrm{C} 17-\mathrm{H} 17 \mathrm{C}$ & 109.5 \\
\hline $\mathrm{C} 16-\mathrm{N} 2-\mathrm{C} 12$ & $110.1(5)$ & $\mathrm{H} 17 \mathrm{~B}-\mathrm{C} 17-\mathrm{H} 17 \mathrm{C}$ & 109.5 \\
\hline $\mathrm{C} 16-\mathrm{N} 2-\mathrm{C} 14$ & $109.8(5)$ & $\mathrm{Br} 2-\mathrm{Pt} 1-\mathrm{Br} 1$ & $91.171(19)$ \\
\hline $\mathrm{N} 2-\mathrm{C} 10-\mathrm{H} 10 \mathrm{~A}$ & 107.9 & $\mathrm{Br} 2-\mathrm{Pt} 1-\mathrm{Br} 3$ & 92.507 (19) \\
\hline $\mathrm{N} 2-\mathrm{C} 10-\mathrm{H} 10 \mathrm{~B}$ & 107.9 & $\mathrm{Br} 3-\mathrm{Pt} 1-\mathrm{Br} 1$ & $176.30(2)$ \\
\hline $\mathrm{H} 10 \mathrm{~A}-\mathrm{C} 10-\mathrm{H} 10 \mathrm{~B}$ & 107.2 & $\mathrm{~N} 1-\mathrm{Pt} 1-\mathrm{Br} 1$ & $87.04(11)$ \\
\hline $\mathrm{C} 11-\mathrm{C} 10-\mathrm{N} 2$ & $117.8(6)$ & $\mathrm{N} 1-\mathrm{Pt} 1-\mathrm{Br} 2$ & $177.41(11)$ \\
\hline $\mathrm{C} 11-\mathrm{C} 10-\mathrm{H} 10 \mathrm{~A}$ & 107.9 & $\mathrm{~N} 1-\mathrm{Pt} 1-\mathrm{Br} 3$ & $89.29(11)$ \\
\hline $\mathrm{C} 11-\mathrm{C} 10-\mathrm{H} 10 \mathrm{~B}$ & 107.9 & $\mathrm{C} 1-\mathrm{S} 1-\mathrm{C} 7$ & $89.9(2)$ \\
\hline $\mathrm{C} 10-\mathrm{C} 11-\mathrm{H} 11 \mathrm{~A}$ & 109.5 & $\mathrm{C} 5-\mathrm{O} 1-\mathrm{C} 9$ & $116.3(5)$ \\
\hline $\mathrm{C} 10-\mathrm{C} 11-\mathrm{H} 11 \mathrm{~B}$ & 109.5 & $\mathrm{C} 1-\mathrm{N} 1-\mathrm{Pt} 1$ & $124.7(3)$ \\
\hline $\mathrm{C} 10-\mathrm{C} 11-\mathrm{H} 11 \mathrm{C}$ & 109.5 & $\mathrm{C} 1-\mathrm{N} 1-\mathrm{C} 2$ & $112.6(4)$ \\
\hline $\mathrm{H} 11 \mathrm{~A}-\mathrm{C} 11-\mathrm{H} 11 \mathrm{~B}$ & 109.5 & $\mathrm{C} 2-\mathrm{N} 1-\mathrm{Pt} 1$ & $122.1(3)$ \\
\hline $\mathrm{H} 11 \mathrm{~A}-\mathrm{C} 11-\mathrm{H} 11 \mathrm{C}$ & 109.5 & $\mathrm{~N} 1-\mathrm{C} 1-\mathrm{S} 1$ & $114.0(4)$ \\
\hline $\mathrm{H} 11 \mathrm{~B}-\mathrm{C} 11-\mathrm{H} 11 \mathrm{C}$ & 109.5 & $\mathrm{~N} 1-\mathrm{C} 1-\mathrm{C} 8$ & $124.2(5)$ \\
\hline
\end{tabular}




\begin{tabular}{|c|c|c|c|}
\hline $\mathrm{N} 2-\mathrm{C} 12-\mathrm{H} 12 \mathrm{~A}$ & 108.0 & $\mathrm{C} 8-\mathrm{C} 1-\mathrm{S} 1$ & $121.8(4)$ \\
\hline $\mathrm{N} 2-\mathrm{C} 12-\mathrm{H} 12 \mathrm{~B}$ & 108.0 & $\mathrm{~N} 1-\mathrm{C} 2-\mathrm{C} 3$ & $126.5(4)$ \\
\hline $\mathrm{H} 12 \mathrm{~A}-\mathrm{C} 12-\mathrm{H} 12 \mathrm{~B}$ & 107.3 & $\mathrm{C} 7-\mathrm{C} 2-\mathrm{N} 1$ & $113.5(4)$ \\
\hline $\mathrm{C} 13-\mathrm{C} 12-\mathrm{N} 2$ & $117.1(6)$ & $\mathrm{C} 7-\mathrm{C} 2-\mathrm{C} 3$ & $120.0(5)$ \\
\hline $\mathrm{C} 13-\mathrm{C} 12-\mathrm{H} 12 \mathrm{~A}$ & 108.0 & $\mathrm{C} 2-\mathrm{C} 3-\mathrm{H} 3$ & 120.9 \\
\hline $\mathrm{C} 13-\mathrm{C} 12-\mathrm{H} 12 \mathrm{~B}$ & 108.0 & $\mathrm{C} 4-\mathrm{C} 3-\mathrm{C} 2$ & $118.2(5)$ \\
\hline $\mathrm{C} 12-\mathrm{C} 13-\mathrm{H} 13 \mathrm{~A}$ & 109.5 & $\mathrm{C} 4-\mathrm{C} 3-\mathrm{H} 3$ & 120.9 \\
\hline $\mathrm{C} 12-\mathrm{C} 13-\mathrm{H} 13 \mathrm{~B}$ & 109.5 & $\mathrm{C} 3-\mathrm{C} 4-\mathrm{H} 4$ & 119.4 \\
\hline $\mathrm{C} 12-\mathrm{C} 13-\mathrm{H} 13 \mathrm{C}$ & 109.5 & $\mathrm{C} 3-\mathrm{C} 4-\mathrm{C} 5$ & $121.2(5)$ \\
\hline $\mathrm{H} 13 \mathrm{~A}-\mathrm{C} 13-\mathrm{H} 13 \mathrm{~B}$ & 109.5 & $\mathrm{C} 5-\mathrm{C} 4-\mathrm{H} 4$ & 119.4 \\
\hline $\mathrm{H} 13 \mathrm{~A}-\mathrm{C} 13-\mathrm{H} 13 \mathrm{C}$ & 109.5 & $\mathrm{O} 1-\mathrm{C} 5-\mathrm{C} 4$ & $115.7(5)$ \\
\hline $\mathrm{H} 13 \mathrm{~B}-\mathrm{C} 13-\mathrm{H} 13 \mathrm{C}$ & 109.5 & $\mathrm{O} 1-\mathrm{C} 5-\mathrm{C} 6$ & $122.6(5)$ \\
\hline $\mathrm{N} 2-\mathrm{C} 14-\mathrm{H} 14 \mathrm{~A}$ & 108.7 & $\mathrm{C} 6-\mathrm{C} 5-\mathrm{C} 4$ & $121.7(5)$ \\
\hline $\mathrm{N} 2-\mathrm{C} 14-\mathrm{H} 14 \mathrm{~B}$ & 108.7 & $\mathrm{C} 5-\mathrm{C} 6-\mathrm{H} 6$ & 121.8 \\
\hline $\mathrm{H} 14 \mathrm{~A}-\mathrm{C} 14-\mathrm{H} 14 \mathrm{~B}$ & 107.6 & $\mathrm{C} 5-\mathrm{C} 6-\mathrm{C} 7$ & $116.5(5)$ \\
\hline $\mathrm{C} 15-\mathrm{C} 14-\mathrm{N} 2$ & $114.3(5)$ & $\mathrm{C} 7-\mathrm{C} 6-\mathrm{H} 6$ & 121.8 \\
\hline $\mathrm{C} 15-\mathrm{C} 14-\mathrm{H} 14 \mathrm{~A}$ & 108.7 & $\mathrm{C} 2-\mathrm{C} 7-\mathrm{S} 1$ & $109.9(4)$ \\
\hline $\mathrm{C} 15-\mathrm{C} 14-\mathrm{H} 14 \mathrm{~B}$ & 108.7 & $\mathrm{C} 2-\mathrm{C} 7-\mathrm{C} 6$ & $122.4(5)$ \\
\hline $\mathrm{C} 14-\mathrm{C} 15-\mathrm{H} 15 \mathrm{~A}$ & 109.5 & $\mathrm{C} 6-\mathrm{C} 7-\mathrm{S} 1$ & $127.7(4)$ \\
\hline $\mathrm{C} 14-\mathrm{C} 15-\mathrm{H} 15 \mathrm{~B}$ & 109.5 & $\mathrm{C} 1-\mathrm{C} 8-\mathrm{H} 8 \mathrm{~A}$ & 109.5 \\
\hline $\mathrm{C} 14-\mathrm{C} 15-\mathrm{H} 15 \mathrm{C}$ & 109.5 & $\mathrm{C} 1-\mathrm{C} 8-\mathrm{H} 8 \mathrm{~B}$ & 109.5 \\
\hline $\mathrm{H} 15 \mathrm{~A}-\mathrm{C} 15-\mathrm{H} 15 \mathrm{~B}$ & 109.5 & $\mathrm{C} 1-\mathrm{C} 8-\mathrm{H} 8 \mathrm{C}$ & 109.5 \\
\hline $\mathrm{H} 15 \mathrm{~A}-\mathrm{C} 15-\mathrm{H} 15 \mathrm{C}$ & 109.5 & $\mathrm{H} 8 \mathrm{~A}-\mathrm{C} 8-\mathrm{H} 8 \mathrm{~B}$ & 109.5 \\
\hline $\mathrm{H} 15 \mathrm{~B}-\mathrm{C} 15-\mathrm{H} 15 \mathrm{C}$ & 109.5 & $\mathrm{H} 8 \mathrm{~A}-\mathrm{C} 8-\mathrm{H} 8 \mathrm{C}$ & 109.5 \\
\hline $\mathrm{N} 2-\mathrm{C} 16-\mathrm{H} 16 \mathrm{~A}$ & 108.4 & $\mathrm{H} 8 \mathrm{~B}-\mathrm{C} 8-\mathrm{H} 8 \mathrm{C}$ & 109.5 \\
\hline $\mathrm{N} 2-\mathrm{C} 16-\mathrm{H} 16 \mathrm{~B}$ & 108.4 & $\mathrm{O} 1-\mathrm{C} 9-\mathrm{H} 9 \mathrm{~A}$ & 109.5 \\
\hline $\mathrm{N} 2-\mathrm{C} 16-\mathrm{C} 17$ & $115.4(5)$ & $\mathrm{O} 1-\mathrm{C} 9-\mathrm{H} 9 \mathrm{~B}$ & 109.5 \\
\hline $\mathrm{H} 16 \mathrm{~A}-\mathrm{C} 16-\mathrm{H} 16 \mathrm{~B}$ & 107.5 & $\mathrm{O} 1-\mathrm{C} 9-\mathrm{H} 9 \mathrm{C}$ & 109.5 \\
\hline $\mathrm{C} 17-\mathrm{C} 16-\mathrm{H} 16 \mathrm{~A}$ & 108.4 & $\mathrm{H} 9 \mathrm{~A}-\mathrm{C} 9-\mathrm{H} 9 \mathrm{~B}$ & 109.5 \\
\hline $\mathrm{C} 17-\mathrm{C} 16-\mathrm{H} 16 \mathrm{~B}$ & 108.4 & $\mathrm{H} 9 \mathrm{~A}-\mathrm{C} 9-\mathrm{H} 9 \mathrm{C}$ & 109.5 \\
\hline $\mathrm{C} 16-\mathrm{C} 17-\mathrm{H} 17 \mathrm{~A}$ & 109.5 & $\mathrm{H} 9 \mathrm{~B}-\mathrm{C} 9-\mathrm{H} 9 \mathrm{C}$ & 109.5 \\
\hline
\end{tabular}

(3) Tetraethylammonium tribromido(2,5,6-trimethyl-1,3-benzothiazole- $\kappa N)$ platinate(II)

Crystal data

$\left(\mathrm{C}_{8} \mathrm{H}_{20} \mathrm{~N}\right)\left[\mathrm{PtBr}_{3}\left(\mathrm{C}_{10} \mathrm{H}_{11} \mathrm{NS}\right)\right]$

$M_{r}=742.33$

Monoclinic, $P 2_{1} / n$

$a=7.9742(4) \AA$

$b=30.2807(14) \AA$

$c=9.6427(5) \AA$

$\beta=100.151(3)^{\circ}$

$V=2291.9(2) \AA^{3}$

$Z=4$
$F(000)=1408$

$D_{\mathrm{x}}=2.151 \mathrm{Mg} \mathrm{m}^{-3}$

Mo $K \alpha$ radiation, $\lambda=0.71073 \AA$

Cell parameters from 5770 reflections

$\theta=2.3-26.4^{\circ}$

$\mu=11.45 \mathrm{~mm}^{-1}$

$T=100 \mathrm{~K}$

Block, red

$0.5 \times 0.36 \times 0.25 \mathrm{~mm}$ 


\section{Data collection}

Bruker APEXII CCD

diffractometer

Radiation source: Micro Focus Rotating Anode, Bruker TXS

Double Bounce Multilayer Mirrors monochromator

Detector resolution: 7.9 pixels $\mathrm{mm}^{-1}$

$\varphi$ and $\omega$ scans

Absorption correction: multi-scan

(SADABS; Bruker, 2014)

\section{Refinement}

Refinement on $F^{2}$

Least-squares matrix: full

$R\left[F^{2}>2 \sigma\left(F^{2}\right)\right]=0.039$

$w R\left(F^{2}\right)=0.106$

$S=1.05$

4692 reflections

266 parameters

0 restraints
$T_{\min }=0.003, T_{\max }=0.028$

10729 measured reflections

4692 independent reflections

4120 reflections with $I>2 \sigma(I)$

$R_{\text {int }}=0.048$

$\theta_{\text {max }}=26.5^{\circ}, \theta_{\text {min }}=2.3^{\circ}$

$h=-9 \rightarrow 5$

$k=-37 \rightarrow 31$

$l=-10 \rightarrow 12$

Special details

Geometry. All e.s.d.'s (except the e.s.d. in the dihedral angle between two 1.s. planes) are estimated using the full covariance matrix. The cell e.s.d.'s are taken into account individually in the estimation of e.s.d.'s in distances, angles and torsion angles; correlations between e.s.d.'s in cell parameters are only used when they are defined by crystal symmetry. An approximate (isotropic) treatment of cell e.s.d.'s is used for estimating e.s.d.'s involving 1.s. planes.

Fractional atomic coordinates and isotropic or equivalent isotropic displacement parameters $\left(\AA^{2}\right)$

\begin{tabular}{llllll}
\hline & $x$ & $y$ & $z$ & $U_{\text {iso }} * U_{\text {eq }}$ & Occ. $(<1)$ \\
\hline N2 & $0.5342(7)$ & $0.18676(17)$ & $0.5028(5)$ & $0.0281(11)$ & \\
C12 & $0.8178(11)$ & $0.1847(4)$ & $0.6760(9)$ & $0.060(2)$ & \\
H12A & 0.8673 & 0.1911 & 0.7743 & $0.089^{*}$ & $0.566(9)$ \\
H12B & 0.8346 & 0.1535 & 0.6560 & $0.089^{*}$ & $0.566(9)$ \\
H12C & 0.8736 & 0.2030 & 0.6137 & $0.089^{*}$ & $0.566(9)$ \\
H12D & 0.9301 & 0.1706 & 0.6901 & $0.089^{*}$ & $0.434(9)$ \\
H12E & 0.8313 & 0.2168 & 0.6706 & $0.089^{*}$ & $0.434(9)$ \\
H12F & 0.7606 & 0.1775 & 0.7551 & $0.089^{*}$ & $0.434(9)$ \\
C14 & $0.4260(12)$ & $0.1207(3)$ & $0.3440(10)$ & $0.057(2)$ & \\
H14A & 0.4285 & 0.0884 & 0.3448 & $0.086^{*}$ & $0.566(9)$ \\
H14B & 0.3076 & 0.1309 & 0.3310 & $0.086^{*}$ & $0.566(9)$ \\
H14C & 0.4792 & 0.1316 & 0.2665 & $0.086^{*}$ & $0.566(9)$ \\
H14D & 0.3786 & 0.1145 & 0.2451 & $0.086^{*}$ & $0.434(9)$ \\
H14E & 0.5321 & 0.1041 & 0.3718 & $0.086^{*}$ & $0.434(9)$ \\
H14F & 0.3439 & 0.1119 & 0.4033 & $0.086^{*}$ & $0.434(9)$ \\
C16 & $0.2437(10)$ & $0.1856(2)$ & $0.5783(8)$ & $0.0404(17)$ & \\
H16A & 0.1334 & 0.2008 & 0.5672 & $0.061^{*}$ & $0.566(9)$ \\
H16B & 0.2262 & 0.1549 & 0.5468 & $0.061^{*}$ & $0.566(9)$ \\
H16C & 0.2981 & 0.1862 & 0.6777 & $0.061^{*}$ & $0.566(9)$ \\
H16D & 0.1836 & 0.1759 & 0.6532 & $0.061^{*}$ & $0.434(9)$
\end{tabular}




\begin{tabular}{|c|c|c|c|c|c|}
\hline $\mathrm{H} 16 \mathrm{E}$ & 0.2345 & 0.2178 & 0.5684 & $0.061 *$ & $0.434(9)$ \\
\hline $\mathrm{H} 16 \mathrm{~F}$ & 0.1925 & 0.1716 & 0.4893 & $0.061^{*}$ & $0.434(9)$ \\
\hline C18 & $0.6616(12)$ & 0.2550 & $0.4092(10)$ & $0.054(2)$ & \\
\hline H18A & 0.7199 & 0.2649 & 0.3334 & $0.081^{*}$ & $0.566(9)$ \\
\hline H18B & 0.5547 & 0.2713 & 0.4045 & $0.081^{*}$ & $0.566(9)$ \\
\hline $\mathrm{H} 18 \mathrm{C}$ & 0.7348 & 0.2603 & 0.5004 & $0.081^{*}$ & $0.566(9)$ \\
\hline H18D & 0.6529 & 0.2872 & 0.4073 & $0.081^{*}$ & $0.434(9)$ \\
\hline H18E & 0.7769 & 0.2463 & 0.4540 & $0.081 *$ & $0.434(9)$ \\
\hline $\mathrm{H} 18 \mathrm{~F}$ & 0.6378 & 0.2435 & 0.3126 & $0.081 *$ & $0.434(9)$ \\
\hline C11 & $0.6256(17)$ & $0.1951(4)$ & $0.6507(12)$ & $0.033(3)$ & $0.566(9)$ \\
\hline H11A & 0.5715 & 0.1771 & 0.7162 & $0.040^{*}$ & $0.566(9)$ \\
\hline H11B & 0.6104 & 0.2266 & 0.6739 & $0.040^{*}$ & $0.566(9)$ \\
\hline C13 & $0.5186(18)$ & $0.1373(4)$ & $0.4765(14)$ & $0.037(3)$ & $0.566(9)$ \\
\hline $\mathrm{H} 13 \mathrm{~A}$ & 0.6356 & 0.1252 & 0.4883 & $0.044^{*}$ & $0.566(9)$ \\
\hline H13B & 0.4650 & 0.1245 & 0.5524 & $0.044^{*}$ & $0.566(9)$ \\
\hline $\mathrm{C} 15$ & $0.3598(15)$ & $0.2095(4)$ & $0.4884(12)$ & $0.032(3)$ & $0.566(9)$ \\
\hline $\mathrm{H} 15 \mathrm{~A}$ & 0.3039 & 0.2093 & 0.3882 & $0.038 *$ & $0.566(9)$ \\
\hline H15B & 0.3758 & 0.2406 & 0.5193 & $0.038 *$ & $0.566(9)$ \\
\hline C17A & $0.6257(15)$ & $0.2086(5)$ & $0.3936(13)$ & $0.040(3)$ & $0.566(9)$ \\
\hline H17A & 0.7349 & 0.1930 & 0.3950 & $0.048^{*}$ & $0.566(9)$ \\
\hline H17B & 0.5555 & 0.2039 & 0.2993 & $0.048 *$ & $0.566(9)$ \\
\hline C11A & $0.716(2)$ & $0.1689(6)$ & $0.546(2)$ & $0.044(5)$ & $0.434(9)$ \\
\hline $\mathrm{H} 11 \mathrm{C}$ & 0.7089 & 0.1363 & 0.5527 & $0.053 *$ & $0.434(9)$ \\
\hline H11D & 0.7792 & 0.1755 & 0.4687 & $0.053 *$ & $0.434(9)$ \\
\hline C13A & $0.460(2)$ & $0.1664(5)$ & $0.3608(15)$ & $0.033(4)$ & $0.434(9)$ \\
\hline $\mathrm{H} 13 \mathrm{C}$ & 0.3509 & 0.1819 & 0.3266 & $0.040 *$ & $0.434(9)$ \\
\hline H13D & 0.5377 & 0.1742 & 0.2953 & $0.040 *$ & $0.434(9)$ \\
\hline $\mathrm{C} 15 \mathrm{~A}$ & $0.435(2)$ & $0.1723(6)$ & $0.6165(14)$ & $0.031(4)$ & $0.434(9)$ \\
\hline $\mathrm{H} 15 \mathrm{C}$ & 0.4863 & 0.1860 & 0.7073 & $0.038 *$ & $0.434(9)$ \\
\hline H15D & 0.4442 & 0.1398 & 0.6281 & $0.038^{*}$ & $0.434(9)$ \\
\hline C17 & $0.535(2)$ & $0.2363(5)$ & $0.4914(17)$ & $0.033(4)$ & $0.434(9)$ \\
\hline $\mathrm{H} 17 \mathrm{C}$ & 0.4195 & 0.2461 & 0.4466 & $0.040^{*}$ & $0.434(9)$ \\
\hline H17D & 0.5582 & 0.2489 & 0.5876 & $0.040^{*}$ & $0.434(9)$ \\
\hline Pt1 & 0.28820 & $0.36639(2)$ & $0.54603(2)$ & $0.02321(10)$ & \\
\hline $\mathrm{Br} 1$ & $0.11056(8)$ & $0.41268(2)$ & $0.37347(7)$ & $0.03333(17)$ & \\
\hline $\mathrm{Br} 2$ & $0.08097(9)$ & $0.30729(2)$ & $0.50744(7)$ & $0.03324(17)$ & \\
\hline $\mathrm{Br} 3$ & $0.47121(9)$ & $0.32318(2)$ & $0.72302(8)$ & $0.04046(19)$ & \\
\hline $\mathrm{S} 1$ & $0.7243(2)$ & $0.46490(5)$ & $0.58781(16)$ & $0.0279(3)$ & \\
\hline N1 & $0.4604(6)$ & $0.41615(16)$ & $0.5825(5)$ & $0.0239(10)$ & \\
\hline $\mathrm{C} 1$ & $0.6036(8)$ & $0.4185(2)$ & $0.5354(6)$ & $0.0271(13)$ & \\
\hline $\mathrm{C} 2$ & $0.4361(8)$ & $0.4523(2)$ & $0.6672(6)$ & $0.0258(13)$ & \\
\hline $\mathrm{C} 3$ & $0.2953(8)$ & 0.4599 (2) & $0.7319(6)$ & $0.0263(13)$ & \\
\hline H3 & 0.2031 & 0.4396 & 0.7202 & $0.032 *$ & \\
\hline $\mathrm{C} 4$ & $0.2917(8)$ & $0.4974(2)$ & $0.8136(6)$ & 0.0285 (13) & \\
\hline $\mathrm{C} 5$ & $0.4298(9)$ & $0.5269(2)$ & $0.8313(7)$ & $0.0319(14)$ & \\
\hline C6 & $0.5667(9)$ & $0.5201(2)$ & $0.7669(6)$ & $0.0294(14)$ & \\
\hline H6 & 0.6578 & 0.5408 & 0.7782 & $0.035^{*}$ & \\
\hline $\mathrm{C} 7$ & $0.5713(8)$ & $0.4824(2)$ & $0.6840(6)$ & $0.0263(13)$ & \\
\hline
\end{tabular}




\begin{tabular}{lllll} 
C8 & $0.6633(9)$ & $0.3844(2)$ & $0.4455(7)$ & $0.0342(15)$ \\
H8A & 0.5737 & 0.3784 & 0.3642 & $0.051^{*}$ \\
H8B & 0.6900 & 0.3572 & 0.5001 & $0.051^{*}$ \\
H8C & 0.7657 & 0.3949 & 0.4126 & $0.051^{*}$ \\
C9 & $0.1406(9)$ & $0.5053(2)$ & $0.8848(7)$ & $0.0346(15)$ \\
H9A & 0.0846 & 0.5330 & 0.8503 & $0.052^{*}$ \\
H9B & 0.1792 & 0.5073 & 0.9869 & $0.052^{*}$ \\
H9C & 0.0599 & 0.4808 & 0.8636 & $0.052^{*}$ \\
C10 & $0.4240(10)$ & $0.5680(2)$ & $0.9223(8)$ & $0.0391(16)$ \\
H10A & 0.3289 & 0.5869 & 0.8798 & $0.059^{*}$ \\
H10B & 0.5311 & 0.5844 & 0.9286 & $0.059^{*}$ \\
H10C & 0.4084 & 0.5591 & 1.0170 & $0.059^{*}$ \\
\hline
\end{tabular}

Atomic displacement parameters $\left(\AA^{2}\right)$

\begin{tabular}{|c|c|c|c|c|c|c|}
\hline & $U^{11}$ & $U^{22}$ & $U^{33}$ & $U^{12}$ & $U^{13}$ & $U^{23}$ \\
\hline N2 & 0.028 (3) & $0.026(3)$ & $0.031(3)$ & $0.001(2)$ & 0.007 (2) & $-0.001(2)$ \\
\hline $\mathrm{C} 12$ & $0.036(4)$ & $0.093(7)$ & $0.047(5)$ & $0.003(4)$ & -0.002 & $0.015(5)$ \\
\hline $\mathrm{C} 14$ & $0.053(5)$ & $0.052(5)$ & $0.072(6)$ & -0.005 (4) & $0.026(5)$ & $-0.035(5)$ \\
\hline $\mathrm{C} 16$ & $0.038(4)$ & $0.037(4)$ & $0.051(4)$ & $0.000(3)$ & $0.023(3)$ & -0.005 \\
\hline $\mathrm{C} 18$ & $0.061(5)$ & $0.045(5)$ & $0.060(5)$ & -0.009 (4) & $0.021(4)$ & $0.014(4)$ \\
\hline $\mathrm{C} 11$ & $0.045(7)$ & $0.027(6)$ & $0.027(5)$ & $-0.004(5)$ & $0.004(5)$ & $-0.001(5)$ \\
\hline C13 & $0.038(7)$ & $0.028(6)$ & $0.043(7)$ & 0.009 (5) & $0.004(6)$ & $-0.008(5)$ \\
\hline $\mathrm{C} 15$ & $0.037(6)$ & $0.023(6)$ & $0.034(6)$ & $0.003(5)$ & $0.007(5)$ & $-0.002(5)$ \\
\hline $\mathrm{C} 17 \mathrm{~A}$ & $0.024(6)$ & $0.063(9)$ & $0.034(6)$ & $0.000(6)$ & $0.010(5)$ & $0.003(6)$ \\
\hline $\mathrm{C} 11 \mathrm{~A}$ & $0.035(9)$ & $0.044(10)$ & $0.058(11)$ & $0.017(8)$ & $0.020(8)$ & $0.022(8)$ \\
\hline $\mathrm{C} 13 \mathrm{~A}$ & $0.049(9)$ & $0.032(8)$ & $0.023(7)$ & $-0.013(7)$ & $0.014(7)$ & $-0.013(6)$ \\
\hline C15A & $0.032(8)$ & 0.044 (9) & $0.020(7)$ & $0.000(7)$ & 0.009 (6) & $0.006(6)$ \\
\hline C17 & $0.037(8)$ & $0.031(8)$ & $0.037(8)$ & $-0.009(7)$ & $0.018(7)$ & $0.001(6)$ \\
\hline Pt1 & $0.02518(15)$ & $0.01967(15)$ & $0.02593(15)$ & $-0.00120(8)$ & $0.00770(10)$ & $0.00056(8)$ \\
\hline $\mathrm{Br} 1$ & $0.0312(3)$ & $0.0284(3)$ & $0.0401(4)$ & $-0.0015(3)$ & $0.0056(3)$ & $0.0073(3)$ \\
\hline $\mathrm{Br} 2$ & 0.0415 (4) & $0.0266(3)$ & $0.0321(3)$ & -0.0074 & 0.0077 (3) & 0.0004 (2) \\
\hline $\mathrm{Br} 3$ & 0.0389 (4) & $0.0386(4)$ & 0.0420 (4) & 0.0009 (3) & $0.0019(3)$ & $0.0113(3)$ \\
\hline S1 & $0.0268(7)$ & $0.0263(8)$ & $0.0320(8)$ & $-0.0029(6)$ & $0.0091(6)$ & $0.0027(6)$ \\
\hline N1 & $0.026(3)$ & $0.025(3)$ & $0.021(2)$ & $0.001(2)$ & $0.006(2)$ & $0.002(2)$ \\
\hline $\mathrm{C} 1$ & $0.030(3)$ & $0.025(3)$ & $0.026(3)$ & -0.004 & $0.006(3)$ & $0.000(2)$ \\
\hline $\mathrm{C} 2$ & 0.023 & $0.027(3)$ & $0.028(3)$ & -0.001 & $0.007(2)$ & $0.006(2)$ \\
\hline $\mathrm{C} 3$ & $0.028(3)$ & $0.022(3)$ & $0.030(3)$ & -0.003 & 0.007 (3) & $0.002(2)$ \\
\hline $\mathrm{C} 4$ & $0.033(3)$ & $0.023(3)$ & $0.031(3)$ & $0.004(3)$ & 0.009 (3) & $-0.001(3)$ \\
\hline $\mathrm{C} 5$ & $0.046(4)$ & $0.025(3)$ & $0.025(3)$ & $0.004(3)$ & $0.005(3)$ & $0.000(2)$ \\
\hline C6 & $0.037(4)$ & $0.021(3)$ & $0.030(3)$ & -0.005 (3) & $0.005(3)$ & $0.005(2)$ \\
\hline $\mathrm{C} 7$ & 0.032 & $0.022(3)$ & $0.026(3)$ & $0.002(2)$ & $0.007(3)$ & $0.007(2)$ \\
\hline $\mathrm{C} 8$ & $0.030(3)$ & 0.037 (4) & 0.039 (4) & $0.001(3)$ & $0.013(3)$ & $-0.005(3)$ \\
\hline C9 & $0.045(4)$ & $0.027(3)$ & $0.034(3)$ & $0.001(3)$ & $0.012(3)$ & $0.001(3)$ \\
\hline C10 & $0.050(4)$ & $0.020(3)$ & 0.048 (4) & $-0.004(3)$ & $0.013(3)$ & $-0.004(3)$ \\
\hline
\end{tabular}


Geometric parameters $\left(\AA,{ }^{\circ}\right)$

\begin{tabular}{|c|c|c|c|}
\hline $\mathrm{N} 2-\mathrm{C} 11$ & $1.504(12)$ & $\mathrm{C} 13-\mathrm{H} 13 \mathrm{~A}$ & 0.9900 \\
\hline $\mathrm{N} 2-\mathrm{C} 13$ & $1.520(12)$ & $\mathrm{C} 13-\mathrm{H} 13 \mathrm{~B}$ & 0.9900 \\
\hline $\mathrm{N} 2-\mathrm{C} 15$ & $1.535(13)$ & $\mathrm{C} 15-\mathrm{H} 15 \mathrm{~A}$ & 0.9900 \\
\hline $\mathrm{N} 2-\mathrm{C} 17 \mathrm{~A}$ & $1.533(13)$ & $\mathrm{C} 15-\mathrm{H} 15 \mathrm{~B}$ & 0.9900 \\
\hline $\mathrm{N} 2-\mathrm{C} 11 \mathrm{~A}$ & $1.537(16)$ & $\mathrm{C} 17 \mathrm{~A}-\mathrm{H} 17 \mathrm{~A}$ & 0.9900 \\
\hline $\mathrm{N} 2-\mathrm{C} 13 \mathrm{~A}$ & $1.523(14)$ & C17A-H17B & 0.9900 \\
\hline $\mathrm{N} 2-\mathrm{C} 15 \mathrm{~A}$ & $1.523(15)$ & $\mathrm{C} 11 \mathrm{~A}-\mathrm{H} 11 \mathrm{C}$ & 0.9900 \\
\hline $\mathrm{N} 2-\mathrm{C} 17$ & $1.505(16)$ & C11A-H11D & 0.9900 \\
\hline $\mathrm{C} 12-\mathrm{H} 12 \mathrm{~A}$ & 0.9800 & $\mathrm{C} 13 \mathrm{~A}-\mathrm{H} 13 \mathrm{C}$ & 0.9900 \\
\hline C12-H12B & 0.9800 & $\mathrm{C} 13 \mathrm{~A}-\mathrm{H} 13 \mathrm{D}$ & 0.9900 \\
\hline $\mathrm{C} 12-\mathrm{H} 12 \mathrm{C}$ & 0.9800 & $\mathrm{C} 15 \mathrm{~A}-\mathrm{H} 15 \mathrm{C}$ & 0.9900 \\
\hline C12-H12D & 0.9800 & $\mathrm{C} 15 \mathrm{~A}-\mathrm{H} 15 \mathrm{D}$ & 0.9900 \\
\hline C12-H12E & 0.9800 & C17-H17C & 0.9900 \\
\hline $\mathrm{C} 12-\mathrm{H} 12 \mathrm{~F}$ & 0.9800 & C17-H17D & 0.9900 \\
\hline $\mathrm{C} 12-\mathrm{C} 11$ & $1.542(15)$ & $\mathrm{Pt} 1-\mathrm{Br} 1$ & $2.4309(7)$ \\
\hline $\mathrm{C} 12-\mathrm{C} 11 \mathrm{~A}$ & $1.45(2)$ & $\mathrm{Pt} 1-\mathrm{Br} 2$ & $2.4198(7)$ \\
\hline $\mathrm{C} 14-\mathrm{H} 14 \mathrm{~A}$ & 0.9800 & $\mathrm{Pt} 1-\mathrm{Br} 3$ & $2.4240(7)$ \\
\hline C14-H14B & 0.9800 & $\mathrm{Pt} 1-\mathrm{N} 1$ & $2.027(5)$ \\
\hline $\mathrm{C} 14-\mathrm{H} 14 \mathrm{C}$ & 0.9800 & $\mathrm{~S} 1-\mathrm{C} 1$ & $1.727(6)$ \\
\hline C14-H14D & 0.9800 & $\mathrm{~S} 1-\mathrm{C} 7$ & $1.739(7)$ \\
\hline C14-H14E & 0.9800 & $\mathrm{~N} 1-\mathrm{C} 1$ & $1.303(8)$ \\
\hline $\mathrm{C} 14-\mathrm{H} 14 \mathrm{~F}$ & 0.9800 & $\mathrm{~N} 1-\mathrm{C} 2$ & $1.401(8)$ \\
\hline $\mathrm{C} 14-\mathrm{C} 13$ & 1.449 (15) & $\mathrm{C} 1-\mathrm{C} 8$ & $1.482(9)$ \\
\hline $\mathrm{C} 14-\mathrm{C} 13 \mathrm{~A}$ & $1.415(16)$ & $\mathrm{C} 2-\mathrm{C} 3$ & $1.397(9)$ \\
\hline C16-H16A & 0.9800 & $\mathrm{C} 2-\mathrm{C} 7$ & $1.398(9)$ \\
\hline C16-H16B & 0.9800 & $\mathrm{C} 3-\mathrm{H} 3$ & 0.9500 \\
\hline $\mathrm{C} 16-\mathrm{H} 16 \mathrm{C}$ & 0.9800 & $\mathrm{C} 3-\mathrm{C} 4$ & $1.385(9)$ \\
\hline C16-H16D & 0.9800 & $\mathrm{C} 4-\mathrm{C} 5$ & $1.404(9)$ \\
\hline C16-H16E & 0.9800 & $\mathrm{C} 4-\mathrm{C} 9$ & $1.507(9)$ \\
\hline $\mathrm{C} 16-\mathrm{H} 16 \mathrm{~F}$ & 0.9800 & $\mathrm{C} 5-\mathrm{C} 6$ & $1.363(10)$ \\
\hline $\mathrm{C} 16-\mathrm{C} 15$ & $1.553(14)$ & $\mathrm{C} 5-\mathrm{C} 10$ & $1.528(9)$ \\
\hline $\mathrm{C} 16-\mathrm{C} 15 \mathrm{~A}$ & $1.561(17)$ & $\mathrm{C} 6-\mathrm{H} 6$ & 0.9500 \\
\hline $\mathrm{C} 18-\mathrm{H} 18 \mathrm{~A}$ & 0.9800 & $\mathrm{C} 6-\mathrm{C} 7$ & $1.400(9)$ \\
\hline C18-H18B & 0.9800 & $\mathrm{C} 8-\mathrm{H} 8 \mathrm{~A}$ & 0.9800 \\
\hline $\mathrm{C} 18-\mathrm{H} 18 \mathrm{C}$ & 0.9800 & $\mathrm{C} 8-\mathrm{H} 8 \mathrm{~B}$ & 0.9800 \\
\hline C18-H18D & 0.9800 & $\mathrm{C} 8-\mathrm{H} 8 \mathrm{C}$ & 0.9800 \\
\hline $\mathrm{C} 18-\mathrm{H} 18 \mathrm{E}$ & 0.9800 & C9-H9A & 0.9800 \\
\hline $\mathrm{C} 18-\mathrm{H} 18 \mathrm{~F}$ & 0.9800 & C9-H9B & 0.9800 \\
\hline $\mathrm{C} 18-\mathrm{C} 17 \mathrm{~A}$ & $1.435(17)$ & C9- $\mathrm{H} 9 \mathrm{C}$ & 0.9800 \\
\hline $\mathrm{C} 18-\mathrm{C} 17$ & $1.500(16)$ & $\mathrm{C} 10-\mathrm{H} 10 \mathrm{~A}$ & 0.9800 \\
\hline $\mathrm{C} 11-\mathrm{H} 11 \mathrm{~A}$ & 0.9900 & $\mathrm{C} 10-\mathrm{H} 10 \mathrm{~B}$ & 0.9800 \\
\hline C11-H11B & 0.9900 & $\mathrm{C} 10-\mathrm{H} 10 \mathrm{C}$ & 0.9800 \\
\hline $\mathrm{C} 11-\mathrm{N} 2-\mathrm{C} 13$ & $109.7(7)$ & $\mathrm{N} 2-\mathrm{C} 17 \mathrm{~A}-\mathrm{H} 17 \mathrm{~B}$ & 107.9 \\
\hline $\mathrm{C} 11-\mathrm{N} 2-\mathrm{C} 15$ & $106.9(8)$ & $\mathrm{C} 18-\mathrm{C} 17 \mathrm{~A}-\mathrm{N} 2$ & $117.4(10)$ \\
\hline $\mathrm{C} 11-\mathrm{N} 2-\mathrm{C} 17 \mathrm{~A}$ & $111.6(8)$ & $\mathrm{C} 18-\mathrm{C} 17 \mathrm{~A}-\mathrm{H} 17 \mathrm{~A}$ & 107.9 \\
\hline
\end{tabular}




\begin{tabular}{|c|c|c|c|}
\hline $\mathrm{C} 13-\mathrm{N} 2-\mathrm{C} 15$ & $112.3(8)$ & $\mathrm{C} 18-\mathrm{C} 17 \mathrm{~A}-\mathrm{H} 17 \mathrm{~B}$ & 107.9 \\
\hline $\mathrm{C} 13-\mathrm{N} 2-\mathrm{C} 17 \mathrm{~A}$ & $110.2(8)$ & $\mathrm{H} 17 \mathrm{~A}-\mathrm{C} 17 \mathrm{~A}-\mathrm{H} 17 \mathrm{~B}$ & 107.2 \\
\hline $\mathrm{C} 17 \mathrm{~A}-\mathrm{N} 2-\mathrm{C} 15$ & $106.1(7)$ & $\mathrm{N} 2-\mathrm{C} 11 \mathrm{~A}-\mathrm{H} 11 \mathrm{C}$ & 107.7 \\
\hline $\mathrm{C} 13 \mathrm{~A}-\mathrm{N} 2-\mathrm{C} 11 \mathrm{~A}$ & $107.5(11)$ & $\mathrm{N} 2-\mathrm{C} 11 \mathrm{~A}-\mathrm{H} 11 \mathrm{D}$ & 107.7 \\
\hline $\mathrm{C} 13 \mathrm{~A}-\mathrm{N} 2-\mathrm{C} 15 \mathrm{~A}$ & $111.2(9)$ & $\mathrm{C} 12-\mathrm{C} 11 \mathrm{~A}-\mathrm{N} 2$ & $118.3(13)$ \\
\hline $\mathrm{C} 15 \mathrm{~A}-\mathrm{N} 2-\mathrm{C} 11 \mathrm{~A}$ & $106.7(9)$ & $\mathrm{C} 12-\mathrm{C} 11 \mathrm{~A}-\mathrm{H} 11 \mathrm{C}$ & 107.7 \\
\hline $\mathrm{C} 17-\mathrm{N} 2-\mathrm{C} 11 \mathrm{~A}$ & $110.9(10)$ & $\mathrm{C} 12-\mathrm{C} 11 \mathrm{~A}-\mathrm{H} 11 \mathrm{D}$ & 107.7 \\
\hline $\mathrm{C} 17-\mathrm{N} 2-\mathrm{C} 13 \mathrm{~A}$ & $110.0(9)$ & $\mathrm{H} 11 \mathrm{C}-\mathrm{C} 11 \mathrm{~A}-\mathrm{H} 11 \mathrm{D}$ & 107.1 \\
\hline $\mathrm{C} 17-\mathrm{N} 2-\mathrm{C} 15 \mathrm{~A}$ & $110.4(9)$ & $\mathrm{N} 2-\mathrm{C} 13 \mathrm{~A}-\mathrm{H} 13 \mathrm{C}$ & 106.7 \\
\hline $\mathrm{H} 12 \mathrm{~A}-\mathrm{C} 12-\mathrm{H} 12 \mathrm{~B}$ & 109.5 & $\mathrm{~N} 2-\mathrm{C} 13 \mathrm{~A}-\mathrm{H} 13 \mathrm{D}$ & 106.7 \\
\hline $\mathrm{H} 12 \mathrm{~A}-\mathrm{C} 12-\mathrm{H} 12 \mathrm{C}$ & 109.5 & $\mathrm{C} 14-\mathrm{C} 13 \mathrm{~A}-\mathrm{N} 2$ & $122.3(12)$ \\
\hline $\mathrm{H} 12 \mathrm{~B}-\mathrm{C} 12-\mathrm{H} 12 \mathrm{C}$ & 109.5 & $\mathrm{C} 14-\mathrm{C} 13 \mathrm{~A}-\mathrm{H} 13 \mathrm{C}$ & 106.7 \\
\hline $\mathrm{H} 12 \mathrm{D}-\mathrm{C} 12-\mathrm{H} 12 \mathrm{E}$ & 109.5 & $\mathrm{C} 14-\mathrm{C} 13 \mathrm{~A}-\mathrm{H} 13 \mathrm{D}$ & 106.7 \\
\hline $\mathrm{H} 12 \mathrm{D}-\mathrm{C} 12-\mathrm{H} 12 \mathrm{~F}$ & 109.5 & $\mathrm{H} 13 \mathrm{C}-\mathrm{C} 13 \mathrm{~A}-\mathrm{H} 13 \mathrm{D}$ & 106.6 \\
\hline $\mathrm{H} 12 \mathrm{E}-\mathrm{C} 12-\mathrm{H} 12 \mathrm{~F}$ & 109.5 & $\mathrm{~N} 2-\mathrm{C} 15 \mathrm{~A}-\mathrm{C} 16$ & $111.4(9)$ \\
\hline $\mathrm{C} 11-\mathrm{C} 12-\mathrm{H} 12 \mathrm{~A}$ & 109.5 & $\mathrm{~N} 2-\mathrm{C} 15 \mathrm{~A}-\mathrm{H} 15 \mathrm{C}$ & 109.4 \\
\hline $\mathrm{C} 11-\mathrm{C} 12-\mathrm{H} 12 \mathrm{~B}$ & 109.5 & $\mathrm{~N} 2-\mathrm{C} 15 \mathrm{~A}-\mathrm{H} 15 \mathrm{D}$ & 109.4 \\
\hline $\mathrm{C} 11-\mathrm{C} 12-\mathrm{H} 12 \mathrm{C}$ & 109.5 & $\mathrm{C} 16-\mathrm{C} 15 \mathrm{~A}-\mathrm{H} 15 \mathrm{C}$ & 109.4 \\
\hline $\mathrm{C} 11 \mathrm{~A}-\mathrm{C} 12-\mathrm{H} 12 \mathrm{D}$ & 109.5 & $\mathrm{C} 16-\mathrm{C} 15 \mathrm{~A}-\mathrm{H} 15 \mathrm{D}$ & 109.4 \\
\hline $\mathrm{C} 11 \mathrm{~A}-\mathrm{C} 12-\mathrm{H} 12 \mathrm{E}$ & 109.5 & $\mathrm{H} 15 \mathrm{C}-\mathrm{C} 15 \mathrm{~A}-\mathrm{H} 15 \mathrm{D}$ & 108.0 \\
\hline $\mathrm{C} 11 \mathrm{~A}-\mathrm{C} 12-\mathrm{H} 12 \mathrm{~F}$ & 109.5 & $\mathrm{~N} 2-\mathrm{C} 17-\mathrm{H} 17 \mathrm{C}$ & 108.5 \\
\hline $\mathrm{H} 14 \mathrm{~A}-\mathrm{C} 14-\mathrm{H} 14 \mathrm{~B}$ & 109.5 & $\mathrm{~N} 2-\mathrm{C} 17-\mathrm{H} 17 \mathrm{D}$ & 108.5 \\
\hline $\mathrm{H} 14 \mathrm{~A}-\mathrm{C} 14-\mathrm{H} 14 \mathrm{C}$ & 109.5 & $\mathrm{C} 18-\mathrm{C} 17-\mathrm{N} 2$ & $115.2(11)$ \\
\hline $\mathrm{H} 14 \mathrm{~B}-\mathrm{C} 14-\mathrm{H} 14 \mathrm{C}$ & 109.5 & $\mathrm{C} 18-\mathrm{C} 17-\mathrm{H} 17 \mathrm{C}$ & 108.5 \\
\hline $\mathrm{H} 14 \mathrm{D}-\mathrm{C} 14-\mathrm{H} 14 \mathrm{E}$ & 109.5 & $\mathrm{C} 18-\mathrm{C} 17-\mathrm{H} 17 \mathrm{D}$ & 108.5 \\
\hline $\mathrm{H} 14 \mathrm{D}-\mathrm{C} 14-\mathrm{H} 14 \mathrm{~F}$ & 109.5 & $\mathrm{H} 17 \mathrm{C}-\mathrm{C} 17-\mathrm{H} 17 \mathrm{D}$ & 107.5 \\
\hline $\mathrm{H} 14 \mathrm{E}-\mathrm{C} 14-\mathrm{H} 14 \mathrm{~F}$ & 109.5 & $\mathrm{Br} 2-\mathrm{Pt} 1-\mathrm{Br} 1$ & $91.23(2)$ \\
\hline $\mathrm{C} 13-\mathrm{C} 14-\mathrm{H} 14 \mathrm{~A}$ & 109.5 & $\mathrm{Br} 2-\mathrm{Pt} 1-\mathrm{Br} 3$ & $91.10(2)$ \\
\hline $\mathrm{C} 13-\mathrm{C} 14-\mathrm{H} 14 \mathrm{~B}$ & 109.5 & $\mathrm{Br} 3-\mathrm{Pt} 1-\mathrm{Br} 1$ & $177.45(3)$ \\
\hline $\mathrm{C} 13-\mathrm{C} 14-\mathrm{H} 14 \mathrm{C}$ & 109.5 & $\mathrm{~N} 1-\mathrm{Pt} 1-\mathrm{Br} 1$ & $89.16(14)$ \\
\hline $\mathrm{C} 13 \mathrm{~A}-\mathrm{C} 14-\mathrm{H} 14 \mathrm{D}$ & 109.5 & $\mathrm{~N} 1-\mathrm{Pt} 1-\mathrm{Br} 2$ & $178.76(14)$ \\
\hline $\mathrm{C} 13 \mathrm{~A}-\mathrm{C} 14-\mathrm{H} 14 \mathrm{E}$ & 109.5 & $\mathrm{~N} 1-\mathrm{Pt} 1-\mathrm{Br} 3$ & $88.50(14)$ \\
\hline $\mathrm{C} 13 \mathrm{~A}-\mathrm{C} 14-\mathrm{H} 14 \mathrm{~F}$ & 109.5 & $\mathrm{C} 1-\mathrm{S} 1-\mathrm{C} 7$ & $89.8(3)$ \\
\hline $\mathrm{H} 16 \mathrm{~A}-\mathrm{C} 16-\mathrm{H} 16 \mathrm{~B}$ & 109.5 & $\mathrm{C} 1-\mathrm{N} 1-\mathrm{Pt} 1$ & $126.1(4)$ \\
\hline $\mathrm{H} 16 \mathrm{~A}-\mathrm{C} 16-\mathrm{H} 16 \mathrm{C}$ & 109.5 & $\mathrm{C} 1-\mathrm{N} 1-\mathrm{C} 2$ & $112.3(5)$ \\
\hline $\mathrm{H} 16 \mathrm{~B}-\mathrm{C} 16-\mathrm{H} 16 \mathrm{C}$ & 109.5 & $\mathrm{C} 2-\mathrm{N} 1-\mathrm{Pt} 1$ & $121.6(4)$ \\
\hline $\mathrm{H} 16 \mathrm{D}-\mathrm{C} 16-\mathrm{H} 16 \mathrm{E}$ & 109.5 & $\mathrm{~N} 1-\mathrm{C} 1-\mathrm{S} 1$ & $114.9(5)$ \\
\hline $\mathrm{H} 16 \mathrm{D}-\mathrm{C} 16-\mathrm{H} 16 \mathrm{~F}$ & 109.5 & $\mathrm{~N} 1-\mathrm{C} 1-\mathrm{C} 8$ & $123.9(6)$ \\
\hline $\mathrm{H} 16 \mathrm{E}-\mathrm{C} 16-\mathrm{H} 16 \mathrm{~F}$ & 109.5 & $\mathrm{C} 8-\mathrm{C} 1-\mathrm{S} 1$ & $121.2(5)$ \\
\hline $\mathrm{C} 15-\mathrm{C} 16-\mathrm{H} 16 \mathrm{~A}$ & 109.5 & $\mathrm{C} 3-\mathrm{C} 2-\mathrm{N} 1$ & $126.5(6)$ \\
\hline $\mathrm{C} 15-\mathrm{C} 16-\mathrm{H} 16 \mathrm{~B}$ & 109.5 & $\mathrm{C} 3-\mathrm{C} 2-\mathrm{C} 7$ & $120.4(6)$ \\
\hline $\mathrm{C} 15-\mathrm{C} 16-\mathrm{H} 16 \mathrm{C}$ & 109.5 & $\mathrm{C} 7-\mathrm{C} 2-\mathrm{N} 1$ & $113.1(5)$ \\
\hline $\mathrm{C} 15 \mathrm{~A}-\mathrm{C} 16-\mathrm{H} 16 \mathrm{D}$ & 109.5 & $\mathrm{C} 2-\mathrm{C} 3-\mathrm{H} 3$ & 120.4 \\
\hline $\mathrm{C} 15 \mathrm{~A}-\mathrm{C} 16-\mathrm{H} 16 \mathrm{E}$ & 109.5 & $\mathrm{C} 4-\mathrm{C} 3-\mathrm{C} 2$ & $119.2(6)$ \\
\hline $\mathrm{C} 15 \mathrm{~A}-\mathrm{C} 16-\mathrm{H} 16 \mathrm{~F}$ & 109.5 & $\mathrm{C} 4-\mathrm{C} 3-\mathrm{H} 3$ & 120.4 \\
\hline $\mathrm{H} 18 \mathrm{~A}-\mathrm{C} 18-\mathrm{H} 18 \mathrm{~B}$ & 109.5 & $\mathrm{C} 3-\mathrm{C} 4-\mathrm{C} 5$ & $119.8(6)$ \\
\hline $\mathrm{H} 18 \mathrm{~A}-\mathrm{C} 18-\mathrm{H} 18 \mathrm{C}$ & 109.5 & $\mathrm{C} 3-\mathrm{C} 4-\mathrm{C} 9$ & $119.1(6)$ \\
\hline $\mathrm{H} 18 \mathrm{~B}-\mathrm{C} 18-\mathrm{H} 18 \mathrm{C}$ & 109.5 & $\mathrm{C} 5-\mathrm{C} 4-\mathrm{C} 9$ & $121.1(6)$ \\
\hline
\end{tabular}




\begin{tabular}{|c|c|c|c|}
\hline $\mathrm{H} 18 \mathrm{D}-\mathrm{C} 18-\mathrm{H} 18 \mathrm{E}$ & 109.5 & $\mathrm{C} 4-\mathrm{C} 5-\mathrm{C} 10$ & $119.0(6)$ \\
\hline $\mathrm{H} 18 \mathrm{D}-\mathrm{C} 18-\mathrm{H} 18 \mathrm{~F}$ & 109.5 & $\mathrm{C} 6-\mathrm{C} 5-\mathrm{C} 4$ & $121.5(6)$ \\
\hline $\mathrm{H} 18 \mathrm{E}-\mathrm{C} 18-\mathrm{H} 18 \mathrm{~F}$ & 109.5 & $\mathrm{C} 6-\mathrm{C} 5-\mathrm{C} 10$ & $119.5(6)$ \\
\hline $\mathrm{C} 17 \mathrm{~A}-\mathrm{C} 18-\mathrm{H} 18 \mathrm{~A}$ & 109.5 & $\mathrm{C} 5-\mathrm{C} 6-\mathrm{H} 6$ & 120.4 \\
\hline $\mathrm{C} 17 \mathrm{~A}-\mathrm{C} 18-\mathrm{H} 18 \mathrm{~B}$ & 109.5 & $\mathrm{C} 5-\mathrm{C} 6-\mathrm{C} 7$ & $119.1(6)$ \\
\hline $\mathrm{C} 17 \mathrm{~A}-\mathrm{C} 18-\mathrm{H} 18 \mathrm{C}$ & 109.5 & $\mathrm{C} 7-\mathrm{C} 6-\mathrm{H} 6$ & 120.4 \\
\hline $\mathrm{C} 17-\mathrm{C} 18-\mathrm{H} 18 \mathrm{D}$ & 109.5 & $\mathrm{C} 2-\mathrm{C} 7-\mathrm{S} 1$ & $109.9(5)$ \\
\hline $\mathrm{C} 17-\mathrm{C} 18-\mathrm{H} 18 \mathrm{E}$ & 109.5 & $\mathrm{C} 2-\mathrm{C} 7-\mathrm{C} 6$ & $120.0(6)$ \\
\hline $\mathrm{C} 17-\mathrm{C} 18-\mathrm{H} 18 \mathrm{~F}$ & 109.5 & $\mathrm{C} 6-\mathrm{C} 7-\mathrm{S} 1$ & $130.1(5)$ \\
\hline $\mathrm{N} 2-\mathrm{C} 11-\mathrm{C} 12$ & $114.7(9)$ & $\mathrm{C} 1-\mathrm{C} 8-\mathrm{H} 8 \mathrm{~A}$ & 109.5 \\
\hline $\mathrm{N} 2-\mathrm{C} 11-\mathrm{H} 11 \mathrm{~A}$ & 108.6 & $\mathrm{C} 1-\mathrm{C} 8-\mathrm{H} 8 \mathrm{~B}$ & 109.5 \\
\hline $\mathrm{N} 2-\mathrm{C} 11-\mathrm{H} 11 \mathrm{~B}$ & 108.6 & $\mathrm{C} 1-\mathrm{C} 8-\mathrm{H} 8 \mathrm{C}$ & 109.5 \\
\hline $\mathrm{C} 12-\mathrm{C} 11-\mathrm{H} 11 \mathrm{~A}$ & 108.6 & $\mathrm{H} 8 \mathrm{~A}-\mathrm{C} 8-\mathrm{H} 8 \mathrm{~B}$ & 109.5 \\
\hline $\mathrm{C} 12-\mathrm{C} 11-\mathrm{H} 11 \mathrm{~B}$ & 108.6 & $\mathrm{H} 8 \mathrm{~A}-\mathrm{C} 8-\mathrm{H} 8 \mathrm{C}$ & 109.5 \\
\hline $\mathrm{H} 11 \mathrm{~A}-\mathrm{C} 11-\mathrm{H} 11 \mathrm{~B}$ & 107.6 & $\mathrm{H} 8 \mathrm{~B}-\mathrm{C} 8-\mathrm{H} 8 \mathrm{C}$ & 109.5 \\
\hline N2-C13-H13A & 107.3 & $\mathrm{C} 4-\mathrm{C} 9-\mathrm{H} 9 \mathrm{~A}$ & 109.5 \\
\hline $\mathrm{N} 2-\mathrm{C} 13-\mathrm{H} 13 \mathrm{~B}$ & 107.3 & $\mathrm{C} 4-\mathrm{C} 9-\mathrm{H} 9 \mathrm{~B}$ & 109.5 \\
\hline $\mathrm{C} 14-\mathrm{C} 13-\mathrm{N} 2$ & $120.2(10)$ & $\mathrm{C} 4-\mathrm{C} 9-\mathrm{H} 9 \mathrm{C}$ & 109.5 \\
\hline $\mathrm{C} 14-\mathrm{C} 13-\mathrm{H} 13 \mathrm{~A}$ & 107.3 & $\mathrm{H} 9 \mathrm{~A}-\mathrm{C} 9-\mathrm{H} 9 \mathrm{~B}$ & 109.5 \\
\hline $\mathrm{C} 14-\mathrm{C} 13-\mathrm{H} 13 \mathrm{~B}$ & 107.3 & $\mathrm{H} 9 \mathrm{~A}-\mathrm{C} 9-\mathrm{H} 9 \mathrm{C}$ & 109.5 \\
\hline $\mathrm{H} 13 \mathrm{~A}-\mathrm{C} 13-\mathrm{H} 13 \mathrm{~B}$ & 106.9 & $\mathrm{H} 9 \mathrm{~B}-\mathrm{C} 9-\mathrm{H} 9 \mathrm{C}$ & 109.5 \\
\hline $\mathrm{N} 2-\mathrm{C} 15-\mathrm{C} 16$ & $111.2(8)$ & $\mathrm{C} 5-\mathrm{C} 10-\mathrm{H} 10 \mathrm{~A}$ & 109.5 \\
\hline $\mathrm{N} 2-\mathrm{C} 15-\mathrm{H} 15 \mathrm{~A}$ & 109.4 & $\mathrm{C} 5-\mathrm{C} 10-\mathrm{H} 10 \mathrm{~B}$ & 109.5 \\
\hline $\mathrm{N} 2-\mathrm{C} 15-\mathrm{H} 15 \mathrm{~B}$ & 109.4 & $\mathrm{C} 5-\mathrm{C} 10-\mathrm{H} 10 \mathrm{C}$ & 109.5 \\
\hline $\mathrm{C} 16-\mathrm{C} 15-\mathrm{H} 15 \mathrm{~A}$ & 109.4 & $\mathrm{H} 10 \mathrm{~A}-\mathrm{C} 10-\mathrm{H} 10 \mathrm{~B}$ & 109.5 \\
\hline $\mathrm{C} 16-\mathrm{C} 15-\mathrm{H} 15 \mathrm{~B}$ & 109.4 & $\mathrm{H} 10 \mathrm{~A}-\mathrm{C} 10-\mathrm{H} 10 \mathrm{C}$ & 109.5 \\
\hline $\mathrm{H} 15 \mathrm{~A}-\mathrm{C} 15-\mathrm{H} 15 \mathrm{~B}$ & 108.0 & $\mathrm{H} 10 \mathrm{~B}-\mathrm{C} 10-\mathrm{H} 10 \mathrm{C}$ & 109.5 \\
\hline $\mathrm{N} 2-\mathrm{C} 17 \mathrm{~A}-\mathrm{H} 17 \mathrm{~A}$ & 107.9 & & \\
\hline
\end{tabular}

(4) Tetraethylammonium tribromido(2-methyl-5-nitro-1,3-benzothiazole- $\kappa \mathrm{N}$ )platinate(II)

Crystal data

$\left(\mathrm{C}_{8} \mathrm{H}_{20} \mathrm{~N}\right)\left[\mathrm{PtBr}_{3}\left(\mathrm{C}_{8} \mathrm{H}_{6} \mathrm{~N}_{2} \mathrm{O}_{2} \mathrm{~S}\right)\right]$

$M_{r}=759.28$

Monoclinic, $P 2{ }_{1} / n$

$a=8.1170(3) \AA$

$b=29.2717(12) \AA$

$c=9.5102(4) \AA$

$\beta=100.720(1)^{\circ}$

$V=2220.17(15) \AA^{3}$

$Z=4$

\section{Data collection}

Bruker APEXII CCD

diffractometer

Radiation source: Micro Focus Rotating Anode, Bruker TXS

Double Bounce Multilayer Mirrors monochromator

Detector resolution: 7.9 pixels $\mathrm{mm}^{-1}$ $\varphi$ and $\omega$ scans
$F(000)=1432$

$D_{\mathrm{x}}=2.272 \mathrm{Mg} \mathrm{m}^{-3}$

Mo $K \alpha$ radiation, $\lambda=0.71073 \AA$

Cell parameters from 9483 reflections

$\theta=2.6-26.4^{\circ}$

$\mu=11.83 \mathrm{~mm}^{-1}$

$T=100 \mathrm{~K}$

Block, bronze

$0.32 \times 0.3 \times 0.25 \mathrm{~mm}$

Absorption correction: multi-scan

(SADABS; Bruker, 2014)

$T_{\min }=0.020, T_{\max }=0.045$

15975 measured reflections

4550 independent reflections

4254 reflections with $I>2 \sigma(I)$

$R_{\text {int }}=0.028$

$\theta_{\max }=26.5^{\circ}, \theta_{\min }=2.3^{\circ}$ 
$h=-10 \rightarrow 10$

$k=-31 \rightarrow 36$

Refinement

Refinement on $F^{2}$

Least-squares matrix: full

$R\left[F^{2}>2 \sigma\left(F^{2}\right)\right]=0.029$

$w R\left(F^{2}\right)=0.060$

$S=1.18$

4550 reflections

240 parameters

0 restraints $l=-11 \rightarrow 7$

Hydrogen site location: inferred from neighbouring sites

$\mathrm{H}$-atom parameters constrained

$w=1 /\left[\sigma^{2}\left(F_{0}{ }^{2}\right)+(0.0044 P)^{2}+13.0832 P\right]$

where $P=\left(F_{\mathrm{o}}{ }^{2}+2 F_{\mathrm{c}}{ }^{2}\right) / 3$

$(\Delta / \sigma)_{\max }=0.002$

$\Delta \rho_{\max }=1.25 \mathrm{e} \AA^{-3}$

$\Delta \rho_{\min }=-1.37$ e $\AA^{-3}$

Special details

Geometry. All e.s.d.'s (except the e.s.d. in the dihedral angle between two 1.s. planes) are estimated using the full covariance matrix. The cell e.s.d.'s are taken into account individually in the estimation of e.s.d.'s in distances, angles and torsion angles; correlations between e.s.d.'s in cell parameters are only used when they are defined by crystal symmetry. An approximate (isotropic) treatment of cell e.s.d.'s is used for estimating e.s.d.'s involving 1.s. planes.

Fractional atomic coordinates and isotropic or equivalent isotropic displacement parameters $\left(\AA^{2}\right)$

\begin{tabular}{lllll}
\hline & $x$ & $y$ & $z$ & $U_{\text {iso }} * U_{\text {eq }}$ \\
\hline N3 & $0.5548(5)$ & $0.31569(15)$ & $0.5135(4)$ & $0.0187(9)$ \\
C9 & $0.3795(6)$ & $0.30025(19)$ & $0.5223(6)$ & $0.0247(12)$ \\
H9A & 0.3850 & 0.2685 & 0.5585 & $0.030^{*}$ \\
H9B & 0.3112 & 0.3000 & 0.4245 & $0.030^{*}$ \\
C10 & $0.2911(7)$ & $0.3294(2)$ & $0.6170(7)$ & $0.0320(13)$ \\
H10A & 0.2786 & 0.3606 & 0.5790 & $0.048^{*}$ \\
H10B & 0.3575 & 0.3300 & 0.7143 & $0.048^{*}$ \\
H10C & 0.1802 & 0.3165 & 0.6191 & $0.048^{*}$ \\
C11 & $0.5608(7)$ & $0.36508(18)$ & $0.4693(6)$ & $0.0257(12)$ \\
H11A & 0.6776 & 0.3727 & 0.4612 & $0.031^{*}$ \\
H11B & 0.5304 & 0.3844 & 0.5459 & $0.031^{*}$ \\
C12 & $0.4466(7)$ & $0.3771(2)$ & $0.3292(6)$ & $0.0281(12)$ \\
H12A & 0.4815 & 0.3601 & 0.2510 & $0.042^{*}$ \\
H12B & 0.4534 & 0.4099 & 0.3115 & $0.042^{*}$ \\
H12C & 0.3308 & 0.3689 & 0.3347 & $0.042^{*}$ \\
C13 & $0.6205(7)$ & $0.28611(19)$ & $0.4041(6)$ & $0.0262(12)$ \\
H13A & 0.5380 & 0.2869 & 0.3133 & $0.031^{*}$ \\
H13B & 0.7258 & 0.2998 & 0.3853 & $0.031^{*}$ \\
C14 & $0.6543(8)$ & $0.2369(2)$ & $0.4470(7)$ & $0.0323(13)$ \\
H14A & 0.6871 & 0.2201 & 0.3675 & $0.048^{*}$ \\
H14B & 0.5527 & 0.2232 & 0.4707 & $0.048^{*}$ \\
H14C & 0.7452 & 0.2354 & 0.5307 & $0.048^{*}$ \\
C15 & $0.6624(7)$ & $0.3097(2)$ & $0.6607(6)$ & $0.0266(12)$ \\
H15A & 0.6503 & 0.2780 & 0.6929 & $0.032^{*}$ \\
H15B & 0.6197 & 0.3303 & 0.7284 & $0.032^{*}$ \\
C16 & $0.8519(7)$ & $0.3199(3)$ & $0.6674(7)$ & $0.0373(15)$ \\
H16A & 0.8974 & 0.2984 & 0.6054 & $0.056^{*}$ \\
H16B & 0.9122 & 0.3164 & 0.7661 & $0.056^{*}$ \\
& & & & \\
\end{tabular}




$\begin{array}{lllll}\text { H16C } & 0.8655 & 0.3512 & 0.6348 & 0.056^{*} \\ \text { Pt1 } & 0.19959(2) & 0.63310(2) & 0.93287(2) & 0.01542(6) \\ \text { Br1 } & 0.01278(6) & 0.67668(2) & 0.75173(6) & 0.02534(12) \\ \text { Br2 } & 0.39391(7) & 0.69652(2) & 0.97693(6) & 0.02338(12) \\ \text { Br3 } & 0.38687(6) & 0.58556(2) & 1.10249(6) & 0.02324(12) \\ \text { S1 } & -0.23297(16) & 0.53228(4) & 0.89580(14) & 0.0211(3) \\ \text { O1 } & 0.4596(5) & 0.50291(14) & 0.6504(4) & 0.0304(9) \\ \text { O2 } & 0.3206(5) & 0.44662(14) & 0.5385(5) & 0.0343(10) \\ \text { N1 } & 0.0320(5) & 0.58046(14) & 0.8995(4) & 0.0168(8) \\ \text { N2 } & 0.3324(6) & 0.48001(15) & 0.6180(5) & 0.0247(10) \\ \text { C1 } & -0.1103(6) & 0.57965(17) & 0.9469(6) & 0.0187(10) \\ \text { C2 } & 0.0503(6) & 0.54253(17) & 0.8172(5) & 0.0187(10) \\ \text { C3 } & 0.1902(6) & 0.53230(17) & 0.7554(5) & 0.0192(10) \\ \text { H3 } & 0.2846 & 0.5520 & 0.7661 & 0.023 * \\ \text { C4 } & 0.1839(7) & 0.49242(17) & 0.6787(6) & 0.0213(11) \\ \text { C5 } & 0.0465(7) & 0.46309(19) & 0.6567(6) & 0.0263(12) \\ \text { H5 } & 0.0473 & 0.4365 & 0.5994 & 0.032^{*} \\ \text { C6 } & -0.0899(7) & 0.47253(18) & 0.7177(6) & 0.0245(12) \\ \text { H6 } & -0.1844 & 0.4528 & 0.7047 & 0.029^{*} \\ \text { C7 } & -0.0852(6) & 0.51233(18) & 0.7997(6) & 0.0212(11) \\ \text { C8 } & -0.1675(7) & 0.61580(18) & 1.0364(6) & 0.0239(11) \\ \text { H8A } & -0.1978 & 0.6433 & 0.9784 & 0.036^{*} \\ \text { H8B } & -0.0770 & 0.6231 & 1.1168 & 0.036^{*} \\ \text { H8C } & -0.2655 & 0.6049 & 1.0732 & 0.036^{*} \\ & & & & \end{array}$

Atomic displacement parameters $\left(\AA^{2}\right)$

\begin{tabular}{lllllll}
\hline & $U^{11}$ & $U^{22}$ & $U^{33}$ & $U^{12}$ & $U^{13}$ & $U^{23}$ \\
\hline $\mathrm{N} 3$ & $0.018(2)$ & $0.024(2)$ & $0.014(2)$ & $0.0008(17)$ & $0.0023(17)$ & $0.0010(17)$ \\
$\mathrm{C} 9$ & $0.020(3)$ & $0.027(3)$ & $0.027(3)$ & $-0.003(2)$ & $0.006(2)$ & $0.004(2)$ \\
$\mathrm{C} 10$ & $0.032(3)$ & $0.036(3)$ & $0.031(3)$ & $0.002(3)$ & $0.013(3)$ & $0.005(3)$ \\
$\mathrm{C} 11$ & $0.025(3)$ & $0.024(3)$ & $0.029(3)$ & $-0.004(2)$ & $0.007(2)$ & $0.002(2)$ \\
$\mathrm{C} 12$ & $0.035(3)$ & $0.028(3)$ & $0.022(3)$ & $0.005(2)$ & $0.009(2)$ & $0.007(2)$ \\
$\mathrm{C} 13$ & $0.030(3)$ & $0.032(3)$ & $0.020(3)$ & $-0.002(2)$ & $0.011(2)$ & $-0.004(2)$ \\
$\mathrm{C} 14$ & $0.034(3)$ & $0.031(3)$ & $0.033(3)$ & $0.011(2)$ & $0.008(3)$ & $-0.004(3)$ \\
$\mathrm{C} 15$ & $0.025(3)$ & $0.033(3)$ & $0.022(3)$ & $0.000(2)$ & $0.002(2)$ & $-0.003(2)$ \\
$\mathrm{C} 16$ & $0.018(3)$ & $0.064(4)$ & $0.026(3)$ & $0.004(3)$ & $-0.006(2)$ & $-0.011(3)$ \\
$\mathrm{P} 1$ & $0.01462(10)$ & $0.01661(9)$ & $0.01517(10)$ & $-0.00278(7)$ & $0.00313(7)$ & $-0.00054(7)$ \\
$\mathrm{Br} 1$ & $0.0203(3)$ & $0.0302(3)$ & $0.0244(3)$ & $-0.0025(2)$ & $0.0012(2)$ & $0.0072(2)$ \\
$\mathrm{B} 2$ & $0.0250(3)$ & $0.0238(3)$ & $0.0213(3)$ & $-0.0058(2)$ & $0.0043(2)$ & $-0.0007(2)$ \\
$\mathrm{Br} 3$ & $0.0194(2)$ & $0.0225(3)$ & $0.0263(3)$ & $-0.0023(2)$ & $-0.0001(2)$ & $0.0038(2)$ \\
$\mathrm{S} 1$ & $0.0183(6)$ & $0.0210(6)$ & $0.0235(7)$ & $-0.0059(5)$ & $0.0029(5)$ & $0.0014(5)$ \\
$\mathrm{O} 1$ & $0.026(2)$ & $0.034(2)$ & $0.032(2)$ & $-0.0023(17)$ & $0.0070(18)$ & $-0.0038(18)$ \\
$\mathrm{O} 2$ & $0.046(3)$ & $0.024(2)$ & $0.037(2)$ & $-0.0025(18)$ & $0.018(2)$ & $-0.0089(18)$ \\
$\mathrm{N} 1$ & $0.014(2)$ & $0.019(2)$ & $0.016(2)$ & $-0.0005(16)$ & $0.0018(17)$ & $0.0022(17)$ \\
$\mathrm{N} 2$ & $0.035(3)$ & $0.019(2)$ & $0.021(2)$ & $0.002(2)$ & $0.008(2)$ & $0.0032(19)$ \\
$\mathrm{C} 1$ & $0.017(2)$ & $0.018(2)$ & $0.022(3)$ & $-0.0015(19)$ & $0.004(2)$ & $0.005(2)$ \\
$\mathrm{C} 2$ & $0.023(3)$ & $0.017(2)$ & $0.014(2)$ & $-0.003(2)$ & $-0.001(2)$ & $0.0027(19)$
\end{tabular}




\begin{tabular}{lllllll} 
C3 & $0.017(2)$ & $0.021(2)$ & $0.018(3)$ & $-0.003(2)$ & $0.000(2)$ & $0.004(2)$ \\
C4 & $0.026(3)$ & $0.019(2)$ & $0.018(3)$ & $0.000(2)$ & $0.002(2)$ & $0.004(2)$ \\
C5 & $0.036(3)$ & $0.020(3)$ & $0.023(3)$ & $-0.006(2)$ & $0.005(2)$ & $-0.001(2)$ \\
C6 & $0.026(3)$ & $0.023(3)$ & $0.022(3)$ & $-0.010(2)$ & $-0.001(2)$ & $0.000(2)$ \\
C7 & $0.022(3)$ & $0.021(3)$ & $0.020(3)$ & $0.000(2)$ & $0.002(2)$ & $0.006(2)$ \\
C8 & $0.020(3)$ & $0.022(3)$ & $0.030(3)$ & $-0.002(2)$ & $0.006(2)$ & $0.000(2)$ \\
\hline
\end{tabular}

Geometric parameters $\left(\AA,{ }^{\circ}\right)$

\begin{tabular}{|c|c|c|c|}
\hline N3-C9 & $1.510(6)$ & C16-H16B & 0.9800 \\
\hline $\mathrm{N} 3-\mathrm{C} 11$ & $1.509(7)$ & $\mathrm{C} 16-\mathrm{H} 16 \mathrm{C}$ & 0.9800 \\
\hline $\mathrm{N} 3-\mathrm{C} 13$ & $1.524(6)$ & $\mathrm{Pt} 1-\mathrm{Br} 1$ & $2.4335(6)$ \\
\hline N3-C15 & $1.516(7)$ & $\mathrm{Pt} 1-\mathrm{Br} 2$ & $2.4216(5)$ \\
\hline C9-H9A & 0.9900 & $\mathrm{Pt} 1-\mathrm{Br} 3$ & $2.4367(5)$ \\
\hline C9-H9B & 0.9900 & $\mathrm{Pt} 1-\mathrm{N} 1$ & $2.041(4)$ \\
\hline $\mathrm{C} 9-\mathrm{C} 10$ & $1.515(8)$ & $\mathrm{S} 1-\mathrm{C} 1$ & $1.724(5)$ \\
\hline $\mathrm{C} 10-\mathrm{H} 10 \mathrm{~A}$ & 0.9800 & $\mathrm{~S} 1-\mathrm{C} 7$ & $1.738(5)$ \\
\hline $\mathrm{C} 10-\mathrm{H} 10 \mathrm{~B}$ & 0.9800 & $\mathrm{O} 1-\mathrm{N} 2$ & $1.221(6)$ \\
\hline $\mathrm{C} 10-\mathrm{H} 10 \mathrm{C}$ & 0.9800 & $\mathrm{O} 2-\mathrm{N} 2$ & $1.228(6)$ \\
\hline $\mathrm{C} 11-\mathrm{H} 11 \mathrm{~A}$ & 0.9900 & $\mathrm{~N} 1-\mathrm{C} 1$ & $1.315(6)$ \\
\hline C11-H11B & 0.9900 & $\mathrm{~N} 1-\mathrm{C} 2$ & $1.383(6)$ \\
\hline $\mathrm{C} 11-\mathrm{C} 12$ & $1.516(8)$ & $\mathrm{N} 2-\mathrm{C} 4$ & $1.476(7)$ \\
\hline $\mathrm{C} 12-\mathrm{H} 12 \mathrm{~A}$ & 0.9800 & $\mathrm{C} 1-\mathrm{C} 8$ & $1.486(7)$ \\
\hline $\mathrm{C} 12-\mathrm{H} 12 \mathrm{~B}$ & 0.9800 & $\mathrm{C} 2-\mathrm{C} 3$ & $1.405(7)$ \\
\hline $\mathrm{C} 12-\mathrm{H} 12 \mathrm{C}$ & 0.9800 & $\mathrm{C} 2-\mathrm{C} 7$ & $1.397(7)$ \\
\hline $\mathrm{C} 13-\mathrm{H} 13 \mathrm{~A}$ & 0.9900 & $\mathrm{C} 3-\mathrm{H} 3$ & 0.9500 \\
\hline C13-H13B & 0.9900 & $\mathrm{C} 3-\mathrm{C} 4$ & $1.372(7)$ \\
\hline $\mathrm{C} 13-\mathrm{C} 14$ & $1.509(8)$ & $\mathrm{C} 4-\mathrm{C} 5$ & $1.392(7)$ \\
\hline $\mathrm{C} 14-\mathrm{H} 14 \mathrm{~A}$ & 0.9800 & $\mathrm{C} 5-\mathrm{H} 5$ & 0.9500 \\
\hline C14-H14B & 0.9800 & $\mathrm{C} 5-\mathrm{C} 6$ & $1.370(8)$ \\
\hline $\mathrm{C} 14-\mathrm{H} 14 \mathrm{C}$ & 0.9800 & C6- $\mathrm{H} 6$ & 0.9500 \\
\hline C15-H15A & 0.9900 & $\mathrm{C} 6-\mathrm{C} 7$ & $1.399(7)$ \\
\hline C15-H15B & 0.9900 & $\mathrm{C} 8-\mathrm{H} 8 \mathrm{~A}$ & 0.9800 \\
\hline $\mathrm{C} 15-\mathrm{C} 16$ & $1.557(8)$ & $\mathrm{C} 8-\mathrm{H} 8 \mathrm{~B}$ & 0.9800 \\
\hline $\mathrm{C} 16-\mathrm{H} 16 \mathrm{~A}$ & 0.9800 & $\mathrm{C} 8-\mathrm{H} 8 \mathrm{C}$ & 0.9800 \\
\hline $\mathrm{C} 9-\mathrm{N} 3-\mathrm{C} 13$ & $108.7(4)$ & $\mathrm{C} 16-\mathrm{C} 15-\mathrm{H} 15 \mathrm{~B}$ & 108.7 \\
\hline $\mathrm{C} 9-\mathrm{N} 3-\mathrm{C} 15$ & $107.5(4)$ & $\mathrm{C} 15-\mathrm{C} 16-\mathrm{H} 16 \mathrm{~A}$ & 109.5 \\
\hline $\mathrm{C} 11-\mathrm{N} 3-\mathrm{C} 9$ & $112.4(4)$ & $\mathrm{C} 15-\mathrm{C} 16-\mathrm{H} 16 \mathrm{~B}$ & 109.5 \\
\hline $\mathrm{C} 11-\mathrm{N} 3-\mathrm{C} 13$ & $108.7(4)$ & $\mathrm{C} 15-\mathrm{C} 16-\mathrm{H} 16 \mathrm{C}$ & 109.5 \\
\hline $\mathrm{C} 11-\mathrm{N} 3-\mathrm{C} 15$ & $108.9(4)$ & $\mathrm{H} 16 \mathrm{~A}-\mathrm{C} 16-\mathrm{H} 16 \mathrm{~B}$ & 109.5 \\
\hline $\mathrm{C} 15-\mathrm{N} 3-\mathrm{C} 13$ & $110.5(4)$ & $\mathrm{H} 16 \mathrm{~A}-\mathrm{C} 16-\mathrm{H} 16 \mathrm{C}$ & 109.5 \\
\hline $\mathrm{N} 3-\mathrm{C} 9-\mathrm{H} 9 \mathrm{~A}$ & 108.6 & $\mathrm{H} 16 \mathrm{~B}-\mathrm{C} 16-\mathrm{H} 16 \mathrm{C}$ & 109.5 \\
\hline N3- C9-H9B & 108.6 & $\mathrm{Br} 1-\mathrm{Pt} 1-\mathrm{Br} 3$ & $176.23(2)$ \\
\hline $\mathrm{N} 3-\mathrm{C} 9-\mathrm{C} 10$ & $114.8(5)$ & $\mathrm{Br} 2-\mathrm{Pt} 1-\mathrm{Br} 1$ & 91.183 (19) \\
\hline $\mathrm{H} 9 \mathrm{~A}-\mathrm{C} 9-\mathrm{H} 9 \mathrm{~B}$ & 107.5 & $\mathrm{Br} 2-\mathrm{Pt} 1-\mathrm{Br} 3$ & 90.989 (19) \\
\hline $\mathrm{C} 10-\mathrm{C} 9-\mathrm{H} 9 \mathrm{~A}$ & 108.6 & $\mathrm{~N} 1-\mathrm{Pt} 1-\mathrm{Br} 1$ & $88.64(11)$ \\
\hline $\mathrm{C} 10-\mathrm{C} 9-\mathrm{H} 9 \mathrm{~B}$ & 108.6 & $\mathrm{~N} 1-\mathrm{Pt} 1-\mathrm{Br} 2$ & $178.40(12)$ \\
\hline
\end{tabular}




\begin{tabular}{|c|c|c|c|}
\hline $\mathrm{C} 9-\mathrm{C} 10-\mathrm{H} 10 \mathrm{~A}$ & 109.5 & $\mathrm{~N} 1-\mathrm{Pt} 1-\mathrm{Br} 3$ & $89.28(11)$ \\
\hline $\mathrm{C} 9-\mathrm{C} 10-\mathrm{H} 10 \mathrm{~B}$ & 109.5 & $\mathrm{C} 1-\mathrm{S} 1-\mathrm{C} 7$ & $90.0(2)$ \\
\hline $\mathrm{C} 9-\mathrm{C} 10-\mathrm{H} 10 \mathrm{C}$ & 109.5 & $\mathrm{C} 1-\mathrm{N} 1-\mathrm{Pt} 1$ & $124.2(3)$ \\
\hline $\mathrm{H} 10 \mathrm{~A}-\mathrm{C} 10-\mathrm{H} 10 \mathrm{~B}$ & 109.5 & $\mathrm{C} 1-\mathrm{N} 1-\mathrm{C} 2$ & $111.9(4)$ \\
\hline $\mathrm{H} 10 \mathrm{~A}-\mathrm{C} 10-\mathrm{H} 10 \mathrm{C}$ & 109.5 & $\mathrm{C} 2-\mathrm{N} 1-\mathrm{Pt} 1$ & $123.8(3)$ \\
\hline $\mathrm{H} 10 \mathrm{~B}-\mathrm{C} 10-\mathrm{H} 10 \mathrm{C}$ & 109.5 & $\mathrm{O} 1-\mathrm{N} 2-\mathrm{O} 2$ & $123.9(5)$ \\
\hline $\mathrm{N} 3-\mathrm{C} 11-\mathrm{H} 11 \mathrm{~A}$ & 108.6 & $\mathrm{O} 1-\mathrm{N} 2-\mathrm{C} 4$ & $118.7(4)$ \\
\hline $\mathrm{N} 3-\mathrm{C} 11-\mathrm{H} 11 \mathrm{~B}$ & 108.6 & $\mathrm{O} 2-\mathrm{N} 2-\mathrm{C} 4$ & $117.4(5)$ \\
\hline $\mathrm{N} 3-\mathrm{C} 11-\mathrm{C} 12$ & $114.9(5)$ & $\mathrm{N} 1-\mathrm{C} 1-\mathrm{S} 1$ & $114.6(4)$ \\
\hline $\mathrm{H} 11 \mathrm{~A}-\mathrm{C} 11-\mathrm{H} 11 \mathrm{~B}$ & 107.5 & $\mathrm{~N} 1-\mathrm{C} 1-\mathrm{C} 8$ & $124.8(5)$ \\
\hline $\mathrm{C} 12-\mathrm{C} 11-\mathrm{H} 11 \mathrm{~A}$ & 108.6 & $\mathrm{C} 8-\mathrm{C} 1-\mathrm{S} 1$ & $120.6(4)$ \\
\hline $\mathrm{C} 12-\mathrm{C} 11-\mathrm{H} 11 \mathrm{~B}$ & 108.6 & $\mathrm{~N} 1-\mathrm{C} 2-\mathrm{C} 3$ & $126.0(5)$ \\
\hline $\mathrm{C} 11-\mathrm{C} 12-\mathrm{H} 12 \mathrm{~A}$ & 109.5 & $\mathrm{~N} 1-\mathrm{C} 2-\mathrm{C} 7$ & $114.2(4)$ \\
\hline $\mathrm{C} 11-\mathrm{C} 12-\mathrm{H} 12 \mathrm{~B}$ & 109.5 & $\mathrm{C} 7-\mathrm{C} 2-\mathrm{C} 3$ & $119.7(5)$ \\
\hline $\mathrm{C} 11-\mathrm{C} 12-\mathrm{H} 12 \mathrm{C}$ & 109.5 & $\mathrm{C} 2-\mathrm{C} 3-\mathrm{H} 3$ & 121.6 \\
\hline $\mathrm{H} 12 \mathrm{~A}-\mathrm{C} 12-\mathrm{H} 12 \mathrm{~B}$ & 109.5 & $\mathrm{C} 4-\mathrm{C} 3-\mathrm{C} 2$ & $116.8(5)$ \\
\hline $\mathrm{H} 12 \mathrm{~A}-\mathrm{C} 12-\mathrm{H} 12 \mathrm{C}$ & 109.5 & $\mathrm{C} 4-\mathrm{C} 3-\mathrm{H} 3$ & 121.6 \\
\hline $\mathrm{H} 12 \mathrm{~B}-\mathrm{C} 12-\mathrm{H} 12 \mathrm{C}$ & 109.5 & $\mathrm{C} 3-\mathrm{C} 4-\mathrm{N} 2$ & $117.7(5)$ \\
\hline $\mathrm{N} 3-\mathrm{C} 13-\mathrm{H} 13 \mathrm{~A}$ & 108.5 & $\mathrm{C} 3-\mathrm{C} 4-\mathrm{C} 5$ & $123.6(5)$ \\
\hline $\mathrm{N} 3-\mathrm{C} 13-\mathrm{H} 13 \mathrm{~B}$ & 108.5 & $\mathrm{C} 5-\mathrm{C} 4-\mathrm{N} 2$ & $118.6(5)$ \\
\hline $\mathrm{H} 13 \mathrm{~A}-\mathrm{C} 13-\mathrm{H} 13 \mathrm{~B}$ & 107.5 & $\mathrm{C} 4-\mathrm{C} 5-\mathrm{H} 5$ & 120.0 \\
\hline $\mathrm{C} 14-\mathrm{C} 13-\mathrm{N} 3$ & $115.3(4)$ & $\mathrm{C} 6-\mathrm{C} 5-\mathrm{C} 4$ & $120.0(5)$ \\
\hline $\mathrm{C} 14-\mathrm{C} 13-\mathrm{H} 13 \mathrm{~A}$ & 108.5 & $\mathrm{C} 6-\mathrm{C} 5-\mathrm{H} 5$ & 120.0 \\
\hline $\mathrm{C} 14-\mathrm{C} 13-\mathrm{H} 13 \mathrm{~B}$ & 108.5 & $\mathrm{C} 5-\mathrm{C} 6-\mathrm{H} 6$ & 121.2 \\
\hline $\mathrm{C} 13-\mathrm{C} 14-\mathrm{H} 14 \mathrm{~A}$ & 109.5 & $\mathrm{C} 5-\mathrm{C} 6-\mathrm{C} 7$ & $117.6(5)$ \\
\hline $\mathrm{C} 13-\mathrm{C} 14-\mathrm{H} 14 \mathrm{~B}$ & 109.5 & $\mathrm{C} 7-\mathrm{C} 6-\mathrm{H} 6$ & 121.2 \\
\hline $\mathrm{C} 13-\mathrm{C} 14-\mathrm{H} 14 \mathrm{C}$ & 109.5 & $\mathrm{C} 2-\mathrm{C} 7-\mathrm{S} 1$ & $109.3(4)$ \\
\hline $\mathrm{H} 14 \mathrm{~A}-\mathrm{C} 14-\mathrm{H} 14 \mathrm{~B}$ & 109.5 & $\mathrm{C} 2-\mathrm{C} 7-\mathrm{C} 6$ & $122.2(5)$ \\
\hline $\mathrm{H} 14 \mathrm{~A}-\mathrm{C} 14-\mathrm{H} 14 \mathrm{C}$ & 109.5 & $\mathrm{C} 6-\mathrm{C} 7-\mathrm{S} 1$ & $128.5(4)$ \\
\hline $\mathrm{H} 14 \mathrm{~B}-\mathrm{C} 14-\mathrm{H} 14 \mathrm{C}$ & 109.5 & $\mathrm{C} 1-\mathrm{C} 8-\mathrm{H} 8 \mathrm{~A}$ & 109.5 \\
\hline N3-C15-H15A & 108.7 & $\mathrm{C} 1-\mathrm{C} 8-\mathrm{H} 8 \mathrm{~B}$ & 109.5 \\
\hline N3-C15-H15B & 108.7 & $\mathrm{C} 1-\mathrm{C} 8-\mathrm{H} 8 \mathrm{C}$ & 109.5 \\
\hline N3-C15-C16 & $114.3(5)$ & $\mathrm{H} 8 \mathrm{~A}-\mathrm{C} 8-\mathrm{H} 8 \mathrm{~B}$ & 109.5 \\
\hline $\mathrm{H} 15 \mathrm{~A}-\mathrm{C} 15-\mathrm{H} 15 \mathrm{~B}$ & 107.6 & $\mathrm{H} 8 \mathrm{~A}-\mathrm{C} 8-\mathrm{H} 8 \mathrm{C}$ & 109.5 \\
\hline $\mathrm{C} 16-\mathrm{C} 15-\mathrm{H} 15 \mathrm{~A}$ & 108.7 & $\mathrm{H} 8 \mathrm{~B}-\mathrm{C} 8-\mathrm{H} 8 \mathrm{C}$ & 109.5 \\
\hline $\mathrm{C} 9-\mathrm{N} 3-\mathrm{C} 11-\mathrm{C} 12$ & $-55.8(6)$ & $\mathrm{N} 1-\mathrm{C} 2-\mathrm{C} 7-\mathrm{S} 1$ & $-2.1(6)$ \\
\hline $\mathrm{C} 9-\mathrm{N} 3-\mathrm{C} 13-\mathrm{C} 14$ & $-68.6(6)$ & $\mathrm{N} 1-\mathrm{C} 2-\mathrm{C} 7-\mathrm{C} 6$ & $178.8(5)$ \\
\hline $\mathrm{C} 9-\mathrm{N} 3-\mathrm{C} 15-\mathrm{C} 16$ & $174.6(5)$ & $\mathrm{N} 2-\mathrm{C} 4-\mathrm{C} 5-\mathrm{C} 6$ & $176.8(5)$ \\
\hline $\mathrm{C} 11-\mathrm{N} 3-\mathrm{C} 9-\mathrm{C} 10$ & $-54.2(6)$ & $\mathrm{C} 1-\mathrm{S} 1-\mathrm{C} 7-\mathrm{C} 2$ & $1.9(4)$ \\
\hline $\mathrm{C} 11-\mathrm{N} 3-\mathrm{C} 13-\mathrm{C} 14$ & $168.7(5)$ & $\mathrm{C} 1-\mathrm{S} 1-\mathrm{C} 7-\mathrm{C} 6$ & $-179.1(5)$ \\
\hline $\mathrm{C} 11-\mathrm{N} 3-\mathrm{C} 15-\mathrm{C} 16$ & $-63.3(6)$ & $\mathrm{C} 1-\mathrm{N} 1-\mathrm{C} 2-\mathrm{C} 3$ & $-177.5(5)$ \\
\hline $\mathrm{C} 13-\mathrm{N} 3-\mathrm{C} 9-\mathrm{C} 10$ & $-174.6(5)$ & $\mathrm{C} 1-\mathrm{N} 1-\mathrm{C} 2-\mathrm{C} 7$ & $1.1(6)$ \\
\hline $\mathrm{C} 13-\mathrm{N} 3-\mathrm{C} 11-\mathrm{C} 12$ & $64.6(6)$ & $\mathrm{C} 2-\mathrm{N} 1-\mathrm{C} 1-\mathrm{S} 1$ & $0.4(6)$ \\
\hline $\mathrm{C} 13-\mathrm{N} 3-\mathrm{C} 15-\mathrm{C} 16$ & $56.0(6)$ & $\mathrm{C} 2-\mathrm{N} 1-\mathrm{C} 1-\mathrm{C} 8$ & $-179.1(5)$ \\
\hline $\mathrm{C} 15-\mathrm{N} 3-\mathrm{C} 9-\mathrm{C} 10$ & $65.7(6)$ & $\mathrm{C} 2-\mathrm{C} 3-\mathrm{C} 4-\mathrm{N} 2$ & $-177.5(4)$ \\
\hline $\mathrm{C} 15-\mathrm{N} 3-\mathrm{C} 11-\mathrm{C} 12$ & $-174.9(4)$ & $\mathrm{C} 2-\mathrm{C} 3-\mathrm{C} 4-\mathrm{C} 5$ & $1.9(8)$ \\
\hline $\mathrm{C} 15-\mathrm{N} 3-\mathrm{C} 13-\mathrm{C} 14$ & $49.2(6)$ & $\mathrm{C} 3-\mathrm{C} 2-\mathrm{C} 7-\mathrm{S} 1$ & $176.6(4)$ \\
\hline
\end{tabular}




$\begin{array}{llll}\mathrm{Pt} 1-\mathrm{N} 1-\mathrm{C} 1-\mathrm{S} 1 & 176.9(2) & \mathrm{C} 3-\mathrm{C} 2-\mathrm{C} 7-\mathrm{C} 6 & -2.4(8) \\ \mathrm{Pt} 1-\mathrm{N} 1-\mathrm{C} 1-\mathrm{C} 8 & -2.6(7) & \mathrm{C} 3-\mathrm{C} 4-\mathrm{C} 5-\mathrm{C} 6 & -2.6(8) \\ \mathrm{Pt} 1-\mathrm{N} 1-\mathrm{C} 2-\mathrm{C} 3 & 6.0(7) & \mathrm{C} 4-\mathrm{C} 5-\mathrm{C} 6-\mathrm{C} 7 & 0.7(8) \\ \mathrm{Pt} 1-\mathrm{N} 1-\mathrm{C} 2-\mathrm{C} 7 & -175.4(3) & \mathrm{C} 5-\mathrm{C} 6-\mathrm{C} 7-\mathrm{S} 1 & 1.7(8) \\ \mathrm{O} 1-\mathrm{N} 2-\mathrm{C} 4-\mathrm{C} 3 & 7.5(7) & \mathrm{C} 5-\mathrm{C} 6-\mathrm{C} 7-\mathrm{C} 2 & -1.4(4) \\ \mathrm{O} 1-\mathrm{N} 2-\mathrm{C} 4-\mathrm{C} 5 & -171.9(5) & \mathrm{C} 7-\mathrm{S} 1-\mathrm{C} 1-\mathrm{N} 1 & 178.2(5) \\ \mathrm{O} 2-\mathrm{N} 2-\mathrm{C} 4-\mathrm{C} 3 & -173.4(5) & \mathrm{C} 7-\mathrm{S} 1-\mathrm{C} 1-\mathrm{C} 8 & 0.6(7) \\ \mathrm{O} 2-\mathrm{N} 2-\mathrm{C} 4-\mathrm{C} 5 & 7.2(7) & \mathrm{C} 7-\mathrm{C} 2-\mathrm{C} 3-\mathrm{C} 4 & \\ \mathrm{~N} 1-\mathrm{C} 2-\mathrm{C} 3-\mathrm{C} 4 & 179.2(5) & & \end{array}$

